2018-04-01

\title{
Simulated Tremor Propagation in the Upper Limb: From Muscle Activity to Joint Displacement
}

Thomas Henry Corie

Brigham Young University

Follow this and additional works at: https://scholarsarchive.byu.edu/etd

Part of the Mechanical Engineering Commons

\section{BYU ScholarsArchive Citation}

Corie, Thomas Henry, "Simulated Tremor Propagation in the Upper Limb: From Muscle Activity to Joint Displacement" (2018). All Theses and Dissertations. 6777.

https://scholarsarchive.byu.edu/etd/6777

This Thesis is brought to you for free and open access by BYU ScholarsArchive. It has been accepted for inclusion in All Theses and Dissertations by an authorized administrator of BYU ScholarsArchive. For more information, please contact scholarsarchive@byu.edu, ellen_amatangelo@byu.edu. 
Simulated Tremor Propagation in the Upper Limb:

From Muscle Activity to Joint Displacement

Thomas Henry Corie

A thesis submitted to the faculty of

Brigham Young University

in partial fulfillment of the requirements for the degree of

Master of Science

Steven K. Charles, Chair

Mark B. Colton

Spencer P. Magleby

Department of Mechanical Engineering

Brigham Young University

Copyright (C) 2018 Thomas Henry Corie

All Rights Reserved 


\author{
ABSTRACT \\ Simulated Tremor Propagation in the Upper Limb: \\ From Muscle Activity to Joint Displacement \\ Thomas Henry Corie \\ Department of Mechanical Engineering, BYU \\ Master of Science
}

Although tremor is the most common movement disorder, there are few non-invasive treatment options. One of the obstacles to creating effective tremor suppression devices is our lack of understanding regarding where tremor originates (which muscles), how it propagates through the limb (to which degrees of freedom, DOF), and where it manifests most severely (which DOF).

To investigate these questions, we created a simple, linear time-invariant model to simulate tremor, with tremorogenic muscle activity input (in the 15 major superficial muscles from the shoulder to the wrist) and joint displacement output (in the 7 major upper limb DOF). The model included excitation-contraction dynamics, musculoskeletal geometry (muscle moment arms) and the mechanical impedance (inertia, damping, and stiffness) of the limb.

From our simulation results, we determined four principles of tremor propagation. First, the distribution of tremor depends strongly on musculoskeletal dynamics. Second, the spreading of tremor is due to inertial coupling (primarily) and musculoskeletal geometry (secondarily). Third, tremor spreads narrowly in the sense that most of the tremor caused by a muscle occurs in a small number of DOF. Lastly, assuming uniform distribution of tremorogenic activity among upper-limb muscles, tremor increases proximal-distally, and the contribution from muscles increases proximal-distally.

Keywords: tremor, tremor characterization, tremor distribution, tremor propagation, upper limb, degrees of freedom 


\section{ACKNOWLEDGEMENTS}

I would like to thank Dr. Charles for all of his hard work and dedication as we finished this project. His direction and example have been invaluable and his kindness was as appreciated as his knowledge. I would also like to thank my committee members, Dr. Colton and Dr.

Magleby, for their help on the project. Thank you Paul, Tim, and my other lab mates for helping with writing and for letting me win at disc golf.

Thank you to the National Institute of Neurological Disorders and Stroke for funding this research.

My research would not have been possible without the support of my wife, Carly, and the happiness brought by our kids, Rosie and Andy.

Most of all, I give thanks to God, who has given me everything good in my life. I have come to know that "all things work together for good to them that love God" (Romans 8:28). 


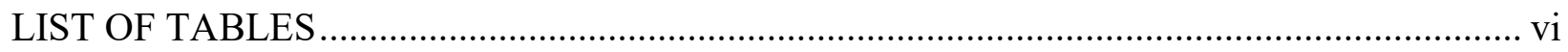

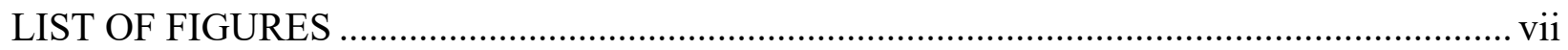

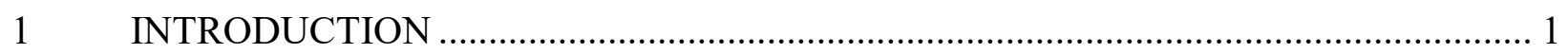

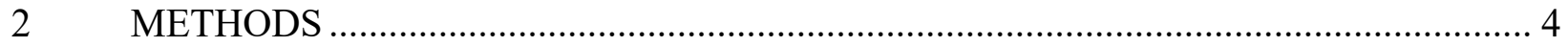

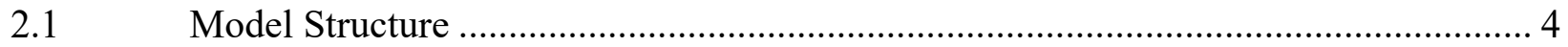

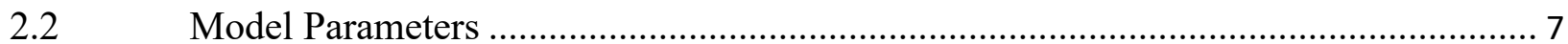

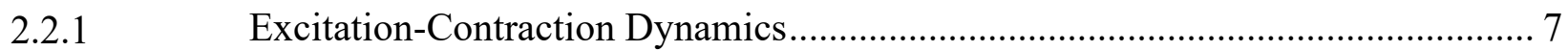

2.2.2 Musculoskeletal Geometry ...................................................................... 9

2.2.3 Mechanical Impedance of the Limb ………………………........................... 9

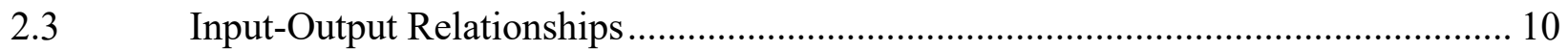

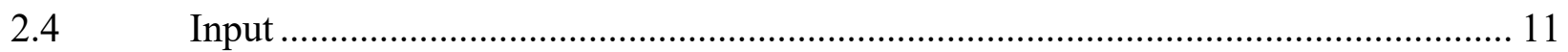

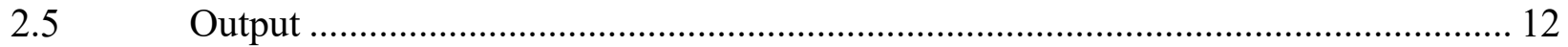

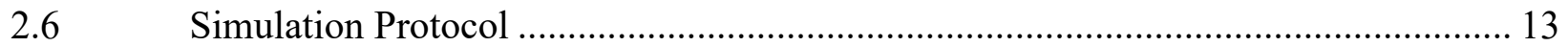

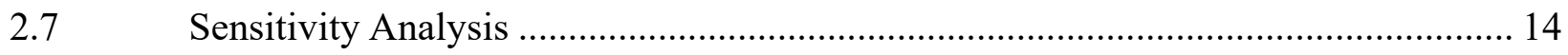

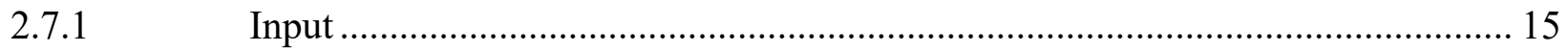

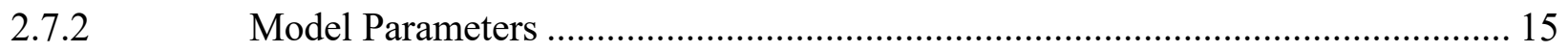

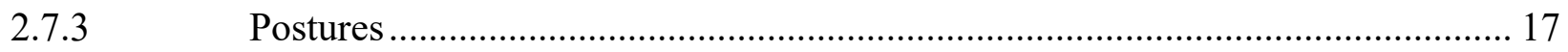

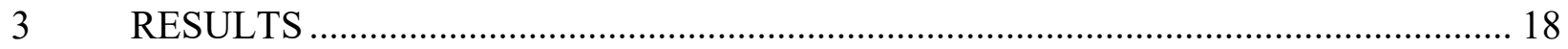

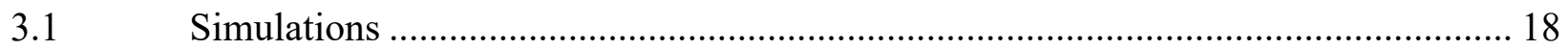

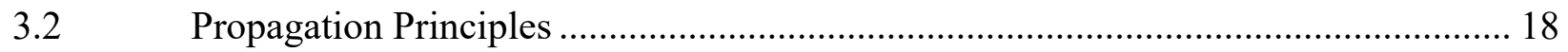

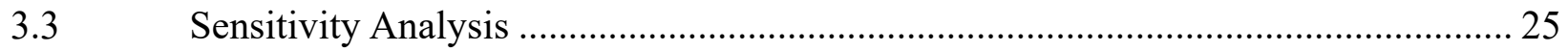

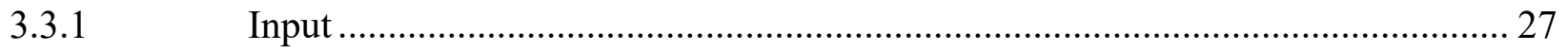

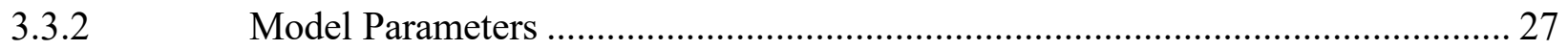




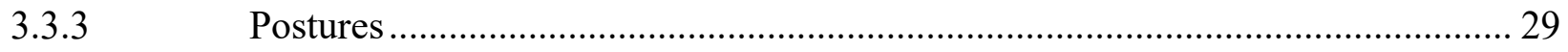

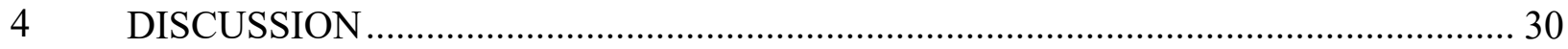

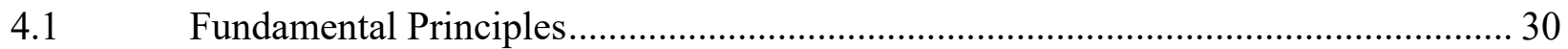

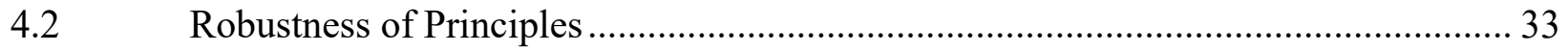

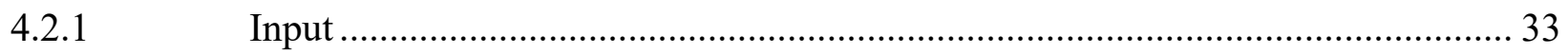

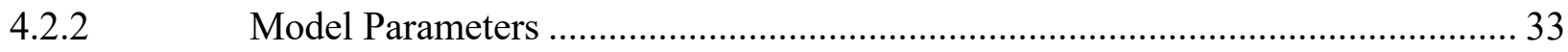

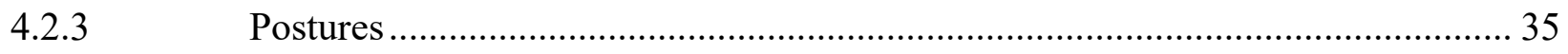

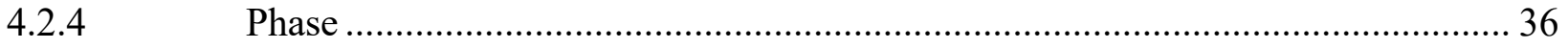

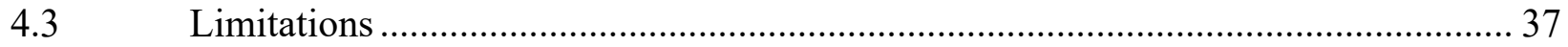

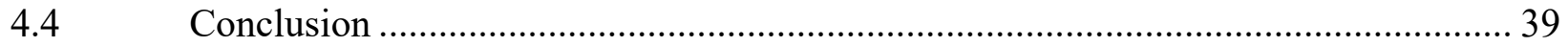

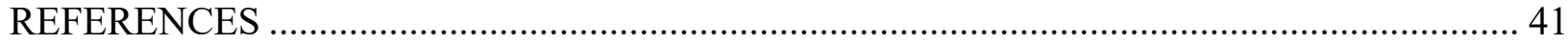

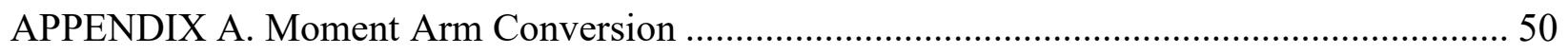

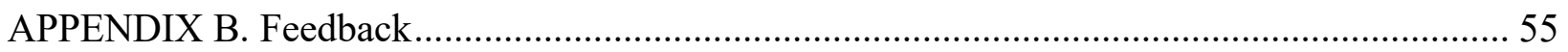




\section{LIST OF TABLES}

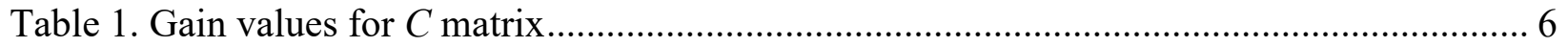

Table 2. Moment arm matrix for Posture 1.............................................................................. 8

Table 3. Heteronymous feedback gain values. ................................................................... 57 


\section{LIST OF FIGURES}

Figure 1. Model of upper limb neuromusculoskeletal dynamics........................................... 5

Figure 2. Input, first sub-model, and Postures .................................................................. 11

Figure 3. System outputs for an input triangle wave ........................................................ 20

Figure 4. Impulse response from all 15 muscles to all 7 DOF. .......................................... 21

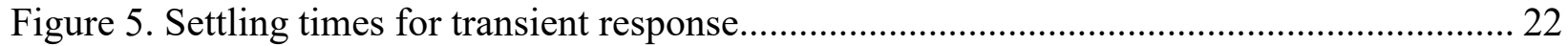

Figure 6. Bode plots for input into each muscle and output in all 7 DOF .............................. 23

Figure 7. Phasor plots grouped according to output DOF .................................................... 24

Figure 8. Graphical representation of magnitude ratios..................................................... 25

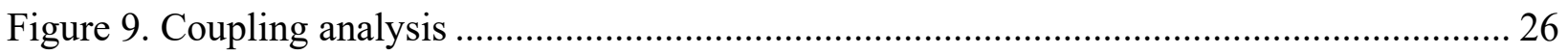

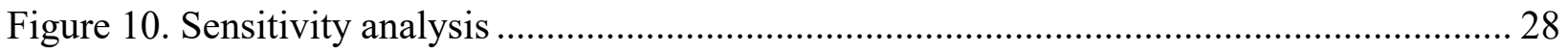

Figure 11. Simulink block diagram for DOF(torque)-level feedback. ................................... 56

Figure 12. Simulink block diagram for muscle-level feedback .......................................... 58 


\section{INTRODUCTION}

Tremor is defined as "an involuntary, rhythmic, oscillatory movement of a body part,"[1] and is the most common movement disorder [2-4]. Essential tremor (ET) alone is estimated to affect 7 million people in the US [5, 6]. Tremor makes activities of daily living (eating, clothing, writing, etc.) difficult or impossible [7, 8].

Although tremor is widespread, current treatment options are unsatisfactory. Present treatments for ET, for example, are limited in their effectiveness. A survey of ET patients found that only one in ten was satisfied with their medical care [9]. The two main treatment options are medication and neurosurgery. The most common medications are $50 \%$ effective, and only in $50 \%$ of patients $[10,11]$; unsurprisingly, many ET patients $(1 / 3$ or more) stop taking their medication when prescribed $[12,13]$.

Surgical treatments (deep brain stimulation, or DBS) have proven more effective (about $90 \%$ tremor reduction [14]), and are effective for a higher percentage of patients $[10,15,16]$. However, because neurosurgery is usually reserved for patients with severe tremor and is highly invasive, it is not an optimal solution in most cases. There is evidence that DBS can cause significant side effects [16-21]. It has been suggested that DBS can lose effectiveness over time [22], and surgical revisions are frequently (greater than 25\%) required [19, 23, 24]. In many cases, those with tremor choose to endure its debilitating effects rather than undertake 
neurosurgery [11-13]; one study found less than 3\% of ET and Parkinson's Disease (PD) patients underwent DBS surgery [25].

These three factors (tremor is very common, medication is only partly effective in only approximately half of patients, and surgery is often undesirable) show the clear need for alternative treatment options. In fact, surveyed ET patients stated that one of the things most lacking in their treatment was an effective, alternative treatment option - something other than medication or surgery [9].

Several tremor-reducing devices have been developed in the past [26-29] but fail to have practical use because of various shortcomings. These devices were novel and well thought-out, but their development would have greatly benefited from a quantitative characterization of tremor. Developing effective devices requires that we understand where the tremor is most severe (which joints), where it originates most commonly (which muscles), how it propagates along the upper limb, and how it can be optimally suppressed. To clarify, by tremor "propagation" we mean the spreading of tremor from tremorogenic activity in a given muscle to tremulous displacement in multiple DOF.

Although tremor affects many degrees of freedom (DOF), past studies of tremor have only focused on local tremor properties. Many studies have investigated aspects of the kinematics and kinetics of tremor, including muscle activity [30-32], ballistic movements [33], eye-hand coordination [34], clinical measures [35-37], and the effect of neural stimulation on tremor [38-40]. However, these studies generally focused on either endpoint tremor or tremor in a single DOF. While simulations of upper limb dynamics and simulations of vibration propagation through structures are commonplace by themselves, no study to date has thoroughly characterized the origin, propagation, and distribution of tremor. 
We built on prior research by Davidson and Charles that modeled tremor in the upper limb from joint torques to joint displacement [41]. The current work expanded the model to begin with muscle activity, which was required to understand how tremor moves from muscle signals to torques at each DOF. The purpose of this work was to establish principles of propagation from tremorogenic ("tremor-generating") muscle activity to hand displacement to determine the feasibility of extracting the origin and propagation of tremor (future work). We anticipate that these principles will enable the development of future treatments that optimally suppress tremor. 


\section{METHODS}

\subsection{Model Structure}

As this is the first simulation of tremor propagation from muscle activity to joint displacement of which we are aware, we deliberately chose a simple model to capture first the most fundamental principles. Our chief objective was to determine fundamental principles of tremor propagation, and to answer the following questions: (1) To what extent do musculoskeletal dynamics affect tremor? (2) Which aspects of the system are most responsible

for spreading the tremor? (3) Does tremor spread broadly from a given muscle to most DOF, or does it spread narrowly, affecting only a small subset of DOF? (4) To which DOF does the input (tremorogenic muscle activity) spread the most? (see Section 2.6).

This model consists of three sub-models that successively transform the muscle activity into muscle force, muscle force into joint torque, and joint torque into joint displacement (Figure 1). As postural tremor consists of relatively small displacements about an equilibrium posture, we used a linear, time-invariant model. It should be noted that performing experiments with tremor patients was not within the scope of this part of our research; the current work lays a foundation for later studies, which will include comparing empirical data to these results.

In all theoretical cases, including in this model, the input is the neural drive to muscle, or muscle activity. As a surrogate for neural drive, we use surface electromyography, sEMG, which is a measured indication of the neural drive. Winter, for example, considered sEMG "a valid 
signal to represent the average motor unit activity of most superficial muscles" [42]. For the sake of consistency, we will refer to the input as muscle activity.

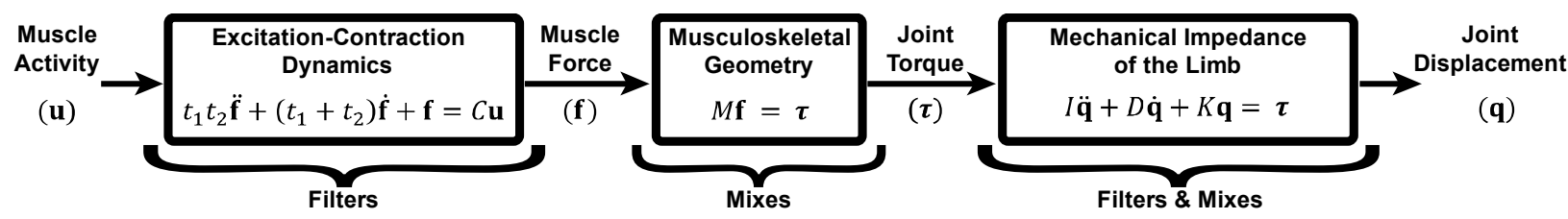

Figure 1. Model of upper limb neuromusculoskeletal dynamics. The sub-models affect tremor by shaping the input through low-pass filtering (Filters) and/or mixing the input into a variety of outputs (Mixes).

The first sub-model, which represents the excitation-contraction coupling dynamics of muscle, transforms muscle activity into muscle force. The 15 muscles (Table 1) were used because they are the major muscles which actuate the 7 DOF from the shoulder to the wrist and they are also superficial (i.e. sEMG sensors can be placed on them). The excitation-contraction coupling dynamics are approximated by a linear, second-order sub-model that has been shown to provide a good prediction of the relationship between EMG and muscle force [42] and has been used successfully to model the control of upper limb movements [43, 44]. This sub-model is defined by time constants representing the dynamics of muscle excitation $\left(t_{1}\right)$ and contraction $\left(t_{2}\right)$ :

$$
t_{1} t_{2} \ddot{\mathbf{f}}+\left(t_{1}+t_{2}\right) \dot{\mathbf{f}}+\mathbf{f}=C \mathbf{u}
$$

where $\mathbf{u}$ is the 15 -element vector of neural input to each of the 15 muscles, $\mathbf{f}$ is the 15 element vector of force produced by each muscle, $t_{1}$ and $t_{2}$ are 15-by-15 diagonal matrices containing the muscle time constants, and $C$ is the 15-by-15 diagonal gain matrix between $\mathbf{u}$ and f. 
Table 1. Gain values for $C$ matrix used to scale the output from the first sub-model. Peak force values were based on values from [45-47].

\begin{tabular}{|c|l|c|c|c|}
\hline $\begin{array}{c}\text { Musc. } \\
\text { No. }\end{array}$ & Muscle & Abbrev. & $\begin{array}{c}\text { Peak } \\
\text { Force (N) }\end{array}$ & $\begin{array}{c}\text { Scaled } \\
\text { Peak Force }\end{array}$ \\
\hline 1 & Anterior Deltoid & DELT1 & 1218.9 & 1.00 \\
\hline 2 & Middle Deltoid & DELT2 & 1103.5 & 0.91 \\
\hline 3 & Posterior Deltoid & DELT3 & 201.6 & 0.17 \\
\hline 4 & Pectoralis Major & PECM2 & 658.3 & 0.54 \\
\hline 5 & Long Head Biceps Brachii & BIClong & 525.1 & 0.43 \\
\hline 6 & Short Head Biceps Brachii & BICshort & 316.8 & 0.26 \\
\hline 7 & Long Head of Triceps Brachii & TRIlong & 771.8 & 0.63 \\
\hline 8 & Lateral Head of Triceps Brachii & TRIlat & 717.5 & 0.59 \\
\hline 9 & Brachialis & BRA & 1177.4 & 0.97 \\
\hline 10 & Brachioradialis & BRD & 276 & 0.23 \\
\hline 11 & Pronator Teres & PT & 557.2 & 0.46 \\
\hline 12 & Flexor Carpi Radialis & FCR & 407.9 & 0.33 \\
\hline 13 & Flexor Carpi Ulnaris & FCU & 479.8 & 0.39 \\
\hline 14 & $\begin{array}{l}\text { Extensor Carpi Radials } \\
\text { (brevis and longus together) }\end{array}$ & $\begin{array}{c}\text { ECRB/ } \\
\text { ECRL }\end{array}$ & 589.8 & 0.48 \\
\hline 15 & Extensor Carpi Ulnaris & ECU & 192.9 & 0.16 \\
\hline
\end{tabular}

The middle sub-model transforms muscle force into joint torques:

$$
M \mathbf{f}=\boldsymbol{\tau}
$$

where $M$ is the 7-by-15 matrix of moment arms (equal to the transpose of the Jacobian from muscle to joint space) and $\boldsymbol{\tau}$ is the 7-element vector of joint torques in the major degrees of freedom from the shoulder to the wrist (positive directions listed first): 1. shoulder flexion/extension (SFE), 2. shoulder adduction/abduction (SAA), 3. shoulder internal rotation (SIER), 4. elbow flexion/extension (EFE), 5. forearm pronation/supination (FPS), 6. wrist flexion/extension (WFE), and 7. wrist ulnar deviation/radial deviation (WRUD). 
The third sub-model transforms joint torques into joint displacements:

$$
I \ddot{\mathbf{q}}+D \dot{\mathbf{q}}+K \mathbf{q}=\boldsymbol{\tau}
$$

where $\boldsymbol{q}$ is the 7-element vector of joint displacements corresponding to $\boldsymbol{\tau}$ (defined above), and $I, D$, and $K$ are 7-by-7 matrices representing the coupled joint inertia, damping, and stiffness in these 7 DOF.

Thus, the entire model transforms the tremorogenic muscle activity in the 15 major superficial muscles from the shoulder to the wrist into tremulous joint displacement in the 7 major DOF actuated by those muscles. It expands the previous investigation of tremor propagation [41], which focused only on the propagation from joint torques to joint displacements (the third sub-model). Note that the second and third sub-models mix the muscle forces into the various joint torques and joint displacements, thereby propagating the tremor, whereas the first sub-model does not mix but simply performs a one-to-one transformation from muscle activity to force within each muscle (Figure 1).

\subsection{Model Parameters}

We took great care to identify physiologically plausible model parameters, as described below. Nevertheless, since we performed an extensive sensitivity analysis (see below), the exact model parameter values are not critical to the conclusions drawn from the simulations.

\subsubsection{Excitation-Contraction Dynamics}

The time constants representing the dynamics of excitation $\left(t_{1}\right)$ and contraction $\left(t_{2}\right)$ depend on the muscle, person, and experimental technique used to measure them [42]. Following [44], we chose default values for $t_{1}$ and $t_{2}$ as $30 \mathrm{~ms}$ and $40 \mathrm{~ms}$, respectively (same for all 
muscles). With these default values, this sub-model acts as an overdamped low-pass filter (cutoff frequency $2.9 \mathrm{~Hz}$ ) with impulse response (representing a muscle twitch) shown in Figure 2C.

Matrix $C$ represents the conversion from steady-state electrical muscle activity to muscle force. In our simulations, we assumed equal input into all muscles (see below) and used $C$ to scale the resulting muscle force according to the maximum force of each muscle (Table 1). Maximum force values were taken from [45-47]. Muscle 14 combines extensor carpi radialis brevis and longus (Table 1), so the peak force in muscle 14 was taken as the sum of the peak forces in each individual muscle.

Because $t_{1}, t_{2}$, and $C$ are diagonal, this sub-model does not propagate (i.e. mix) tremor between muscles but simply transforms muscle activity into force within each muscle. Since the default values for $t_{1}$ and $t_{2}$ are the same for all muscles, but the diagonal values of $C$ depend on the maximum force of each muscle, the impulse responses of the different muscles are simply scaled versions of each other (and of the response shown in Figure 2C).

Table 2. Moment arm matrix for Posture 1. Each column represents the moment arm for a given muscle; rows represent data for each DOF. Shaded values indicate the largest magnitude for a given muscle; these values were retained for coupling analysis. For an arm model representing a $10^{\text {th }}$ or $90^{\text {th }}$ percentile male (by height), * indicates $1-5 \%$ change, $\uparrow=8-11 \%$ change, $\$=23-36 \%$ change.

\begin{tabular}{|c|c|c|c|c|c|c|c|c|c|c|c|c|c|c|c|}
\cline { 2 - 15 } \multicolumn{1}{c|}{} & DELT1 & DELT2 & DELT3 & PECM2 & BIClong & BICshort & TRIlong & TRIlat & BRA & BRD & PT & FCR & FCU & $\begin{array}{c}\text { ECRB/ } \\
\text { ECRL }\end{array}$ & ECU \\
\hline SFE & 0 & 0 & 0 & 0 & 0 & 0 & 0 & 0 & 0 & 0 & 0 & 0 & 0 & 0 & 0 \\
\hline SAA & $-15.5^{*}$ & $34.1^{*}$ & $17.9^{*}$ & $-56.5^{*}$ & $5.33 \dagger$ & $-30.7^{*}$ & -6.93 & 0 & 0 & 0 & 0 & 0 & 0 & 0 & 0 \\
\hline SIER & $5.08^{*}$ & $1.88^{*}$ & $-8.58^{*}$ & $9.61 \dagger$ & 5.84 & $4.19^{*}$ & -4.88 & 0 & 0 & 0 & 0 & 0 & 0 & 0 & 0 \\
\hline EFE & 0 & 0 & 0 & 0 & $45.8 \dagger$ & $45.8 \dagger$ & $-19.6 \dagger$ & $-19.6 \dagger$ & $22.7 \dagger$ & $78.6 \dagger$ & $14.5 \dagger$ & $13.0 \dagger$ & $13.6 \dagger$ & $13.1 \dagger$ & $-2.66 \dagger$ \\
\hline FPS & 0 & 0 & 0 & 0 & $-12.8 \dagger$ & $-12.8 \dagger$ & 0 & 0 & 0 & $5.09 \dagger$ & $9.95 \dagger$ & $1.97 \dagger$ & $1.17 \dagger$ & $-0.22 \dagger$ & $-0.69 \dagger$ \\
\hline WFE & 0 & 0 & 0 & 0 & 0 & 0 & 0 & 0 & 0 & 0 & 0 & $14.9 *$ & $14.9 *$ & $-11.5 *$ & $-6.30 \dagger$ \\
\hline WRUD & 0 & 0 & 0 & 0 & 0 & 0 & 0 & 0 & 0 & 0 & 0 & $-7.47 *$ & $21.8^{*}$ & $-17.3^{*}$ & $25.1 *$ \\
\hline
\end{tabular}




\subsubsection{Musculoskeletal Geometry}

The moment arm matrix, $M$ (Table 2), was determined from OpenSim [48] using a dynamic model of the upper limb "designed to represent the anthropometry and muscle forcegenerating characteristics of a 50th percentile adult male" [47]. OpenSim follows the ISB convention for joint angles [49], which specifies a $Y X Y$ Euler angle sequence [47] for the shoulder. As our model used a $Z X Y$ Euler angle sequence at the shoulder, $M$ was transformed from $Y X Y$ to $Z X Y$ before being implemented in our model (see APPENDIX A). For muscle 14, Extensor Carpi Radialis (brevis and longus together), we used the average of the moment arms for extensor carpi radialis longus and extensor carpi radialis brevis. The moment arms are configuration-dependent, so we calculated a different $M$ for each posture. Since we only looked at postural tremor, which involves relatively small displacements from the reference posture, we left $M$ constant in a given posture.

\subsubsection{Mechanical Impedance of the Limb}

Matrices $I, D$, and $K$ are 7-by-7 impedance matrices representing inertia, damping, and stiffness, respectively. The default values of $I, D$, and $K$ were obtained from the literature [50-53] and are described in detail in [41]. Summarizing, $I$ was calculated from the inertia values of individual limb segments for an average young adult male [53] using the Robotics, Vision and Control (RVC) toolbox in Matlab [54]. As for $M$, we calculated the $I$ matrix for each posture but left it constant in a given posture since tremulous displacements about that posture are relatively small. The default values for $D$ and $K$ represented average joint damping and stiffness of the passive limb (i.e. in the absence of muscle contraction), but we included in the sensitivity analysis simulations of active damping and stiffness as well. The diagonal elements of the impedance matrices represent the relationship between torque applied in a DOF and the resulting 
displacement in that DOF, whereas the off-diagonal elements represent mechanical coupling, i.e. the relationship between torque applied in a DOF and the resulting displacement in other DOF.

\subsection{Input-Output Relationships}

Our full model has 15 inputs (muscle activity in each of the 15 muscles) and 7 outputs (displacement in each of the 7 DOF). Since the model is linear, the relationship between each input and each output is fully described by the transfer function associated with that input and output. For our model, this means the response of the whole system can be described by a 7-by15 matrix of transfer functions, derived as follows. In the Laplace domain, the three sub-models can be expressed as $\boldsymbol{F}=G_{1} \boldsymbol{U}, \boldsymbol{T}=G_{2} \boldsymbol{F}$, and $\boldsymbol{Q}=G_{3} \boldsymbol{T}$, where $\boldsymbol{F}, \boldsymbol{U}, \boldsymbol{T}$, and $\boldsymbol{Q}$ are the Laplace transforms of $\mathbf{f}, \mathbf{u}, \boldsymbol{\tau}$, and $\mathbf{q}$, respectively, and $G_{1}=\left[t_{1} t_{2} s^{2}+\left(t_{1}+t_{2}\right) s+L\right]^{-1} C, G_{2}=M$, and $G_{3}=\left(I s^{2}+D s+K\right)^{-1}$. Variable $s$ is the Laplace variable and $L$ is the 15 -by-15 identity matrix. Combining these sub-models yields $\boldsymbol{Q}=G \boldsymbol{U}$, where $G=G_{1} G_{2} G_{3}$ is the 7-by-15 matrix of transfer functions relating each input in $\boldsymbol{U}$ to each output in $\boldsymbol{Q}$. Therefore, the output in DOF $i$ due to an input in muscle $k$ is $Q_{i / k}=G_{i k} U_{k}$, where $G_{i k}$ is the transfer function in row $i$ and column $k$ of $G$. The total output in DOF $i$ is the linear combination of the inputs in all 15 muscles, the weights of the linear combination being the transfer functions associated with that DOF (row $i$ of $G$ ): $Q_{i}=\sum_{k=1}^{15} G_{i k} U_{k}$. 

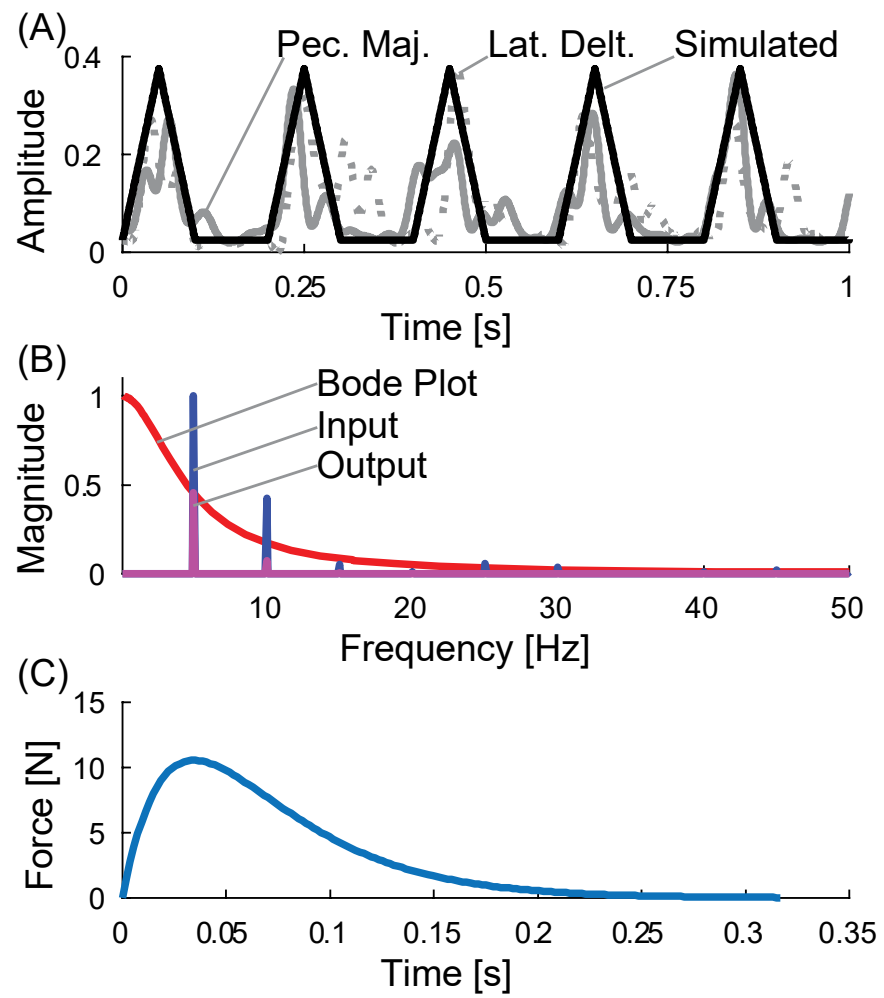

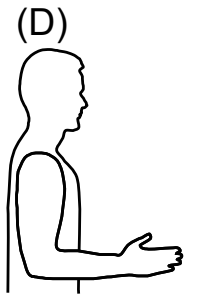

Posture 1

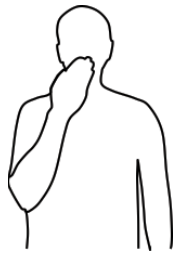

Posture 2

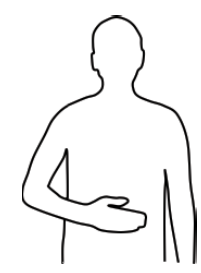

Posture 3

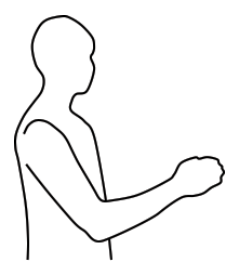

Posture 4

Figure 2. (A) Simulated triangle wave compared to rectified, low-pass filtered EMG data (recorded in $\mathrm{mV}$ ) from a subject with severe ET performing a postural task. The two muscles' data were aligned to be in phase with each other. (B) Bode plot for first sub-model, excitationcontraction dynamics (using default values and $C=1$ ), with the Fourier plot of input signal, $\mathbf{u}$, and output, f, for a $110 \mathrm{~ms}$ wide, $5 \mathrm{~Hz}$ triangle wave input. (C) Impulse response for excitationcontraction dynamics (using default values and $C=1$ ), which represents a muscle twitch. (D) Different postures. Posture 2: hand in front of mouth, representing feeding and grooming activities; Posture 3: hand in workspace in front of abdomen, representing many activities of daily living; Posture 4: arm somewhat outstretched, representing reaching. Joint angles for each posture are given in [41].

\subsection{Input}

The input, $\mathbf{u}$, was based on past studies $[55,56]$ and unpublished data from our lab.

According to these sources, the envelope of (detrended and rectified) tremogenic muscle activity 
can be approximated as a train of triangular pulses separated by periods of no activity (Figure 2A). The duration between pulses is the period of the tremor and thus depends on the tremor frequency, but the mean width of the triangular pulses was 110-120 ms (range 70-160ms) [56], which is within the 50-200 ms "EMG burst duration" range in [57] (see also [58, 59]). We investigated tremor frequencies in the 4-12 Hz tremor band where tremor usually resides [1, 4] and chose the default width of the triangular pulses to be $110 \mathrm{~ms}$. That said, the sensitivity analysis (below) revealed tremor propagation to be quite insensitive not only to the width of the triangular pulses, but even to the shape of the pulses.

\subsection{Output}

The response of a stable, linear system to a periodic input is comprised of the transient response, which decays and disappears with time, and the steady-state response, which remains while the input is applied [60]. We used Matlab functions impulse and stepinfo to characterize the transient response of the system (for all 105 transfer functions). Most of our investigation, however, focused on the steady-state response, which we analyzed as follows. The steady-state response of a linear system to a periodic input is characterized by the frequency response of the system at the frequencies of the input $[41,60]$. The input, approximated as a train of triangular pulses, contains power at the frequency at which the pulses repeat (i.e. the fundamental frequency of the tremor) and at higher harmonics. However, because the full model is a low-pass filter, the harmonics are strongly suppressed, resulting in an output that is practically indistinguishable from a pure sinusoid at the fundamental tremor frequency (Figure 2B, see also Section 3.3.1). Therefore, for practical purposes, the frequency response of the system is fully characterized by the frequency response of the system at the fundamental frequency (no need to 
include the frequency response at harmonic frequencies), allowing us to focus on the frequency response in the $4-12 \mathrm{~Hz}$ tremor band.

\subsection{Simulation Protocol}

In a previous publication that focused only on the propagation from joint torque to joint displacement (the third sub-model in Figure 1), we established six principles of tremor propagation [41]. The main goal of the current study was to determine equivalent principles for the propagation of tremor from muscle activity to joint displacement (the full model in Figure 1). To this end, we determined the extent to which the original principles (established for the third sub-model) held true for the full model. More specifically, we investigated the following questions.

(1) To what extent do musculoskeletal dynamics affect tremor? Musculoskeletal dynamics have the potential to affect tremor in two ways: by 1) shaping the input through lowpass filtering and 2) mixing the input into a variety of outputs. To investigate the amount of shaping and mixing, we used Matlab's bode function to calculate the magnitude ratio and phase shift of all 105 input-output relationships in the $4-12 \mathrm{~Hz}$ tremor band.

(2) Which aspects of the system are most responsible for spreading the tremor? As explained above, the first sub-model is incapable of spreading tremor, but the second sub-model spreads tremor from force in a given muscle to joint torque in multiple DOF through the moment arm matrix $M$. The third sub-model spreads tremor from joint torque in a given DOF to joint displacement in multiple DOF through the coupling terms of the impedance matrices $I, D$, and $K$. However, in a previous investigation of spreading that focused only on the third sub-model, we determined that most of the spreading was due to $I$, and that $D$ and $K$ contributed very little [41]. Therefore, we focused here on the relative contributions of $M$ vs. $I$ in spreading tremor. To 
determine how much of the spreading came from $M$ vs. $I$, we compared the output from the default model to the output from two partially uncoupled models. In the first model, $M$ was altered so it transformed muscle force to joint torque in only one DOF (the DOF with the largest moment arm), reducing $M$ to a "quasi-diagonal" matrix with only one non-zero value per column (Table 2). In the second model, I was diagonalized to remove all coupling terms. Comparing the difference between the outputs of the default model and each adjusted model allowed us to determine the contribution of $M$ vs. $I$.

(3) Does tremor spread broadly from a given muscle to most DOF, or does it spread narrowly, affecting only a small subset of DOF? To answer this question, we used phasor plots to compare the magnitudes of the outputs from a given muscle and determine if outputs in one or two DOF dominated over the outputs in the other DOF.

(4) To which DOF does the input (tremorogenic muscle activity) spread the most?

Assuming equal input into all muscles, we compared the magnitude of the total output tremor between DOF.

\subsection{Sensitivity Analysis}

We performed a sensitivity analysis to evaluate the effect of inaccuracies in our model parameters and simulate differences between subjects. Our main goal was to assess the effect of changes in model parameters on our conclusions to determine if our conclusions were robust. To this end, we varied the input, each of the model parameters $\left(t_{1}, t_{2}, C, M, I, D\right.$, and $\left.K\right)$, and the posture of the limb and determined the sensitivity of our conclusions to these changes. 


\subsubsection{Input}

We varied the width of the triangular pulse from 50 to $200 \mathrm{~ms}$. This range is the range of "EMG burst duration" measured in [57] and is larger than the range of triangular pulse widths (70-160ms) observed in [56]. Furthermore, we assessed the effect of input shape by repeating our simulations with a variety of input shapes. The shapes included a train of narrow (20 ms wide) rectangle waves to approximate impulses, a simple squared sine wave (made with $\left[\sin \left(2 \pi\left(\frac{f}{2}\right) t\right)\right]^{2}$ ), and a squared sine wave with two frequencies (made with $\left.\frac{1}{2}\left[\sin \left(2 \pi\left(\frac{f}{2}\right) t\right)\right]^{2}+\frac{1}{2}\left[\sin \left(2 \pi\left(\frac{3 f}{2}\right) t\right)\right]^{2}\right)$. In each test, the driving frequency $(f$, in $\mathrm{Hz})$ was the same and we tested through the tremor band. The squared sine wave was made because [61] used a squared sine wave to estimate ET patient data; we wanted to determine if the triangle wave, which has the advantage of being able to dictate the width, was significantly different because of the sharper (less smooth) shape. The squared sine wave with two frequencies was tested because actual patient data often had secondary spikes, and we wished to find out if having a second frequency at a smaller magnitude had any significant effect.

\subsubsection{Model Parameters}

Sub-model 1: Time constants $t_{1}$ and $t_{2}$ represent the dynamics of muscle excitation and contraction, respectively. To simulate a generous range of variability between subjects, we halved and doubled the default values (30 and $40 \mathrm{~ms}$ ), resulting in four simulations: with $t_{1}$ at 15 and $30 \mathrm{~ms}$ (with $t_{2} \mathrm{kept}$ at $40 \mathrm{~ms}$ ) and with $t_{2}$ at $80 \mathrm{~ms}$ (with $t_{1}$ at 15 and $30 \mathrm{~ms}$ ). The muscle model is a second-order model with two real, distinct roots $\left(\frac{1}{t_{1}}\right.$ and $\left.\frac{1}{t_{2}}\right)$. Therefore, it suffices to change only one or both of them, but no new information is gained by changing each individually. Varying the time constants over this range varied the low-pass filter cut-off 
frequency of sub-model 1 from 1.8-3.5 Hz. As mentioned above, varying individual diagonal elements of $C$ simply scales the magnitude of the response in the DOF associated with that element. To test the robustness of Principle 4, we scaled the individual entries in $C$ for the most proximal muscles (1-4) in order to determine what conditions would lead to increased tremor in the proximal DOF.

Sub-model 2: The moment arm matrix, $M$, was varied by scaling the upper-limb model in OpenSim. More specifically, we repeated our simulations with $M$ for a $10^{\text {th }}$ and $90^{\text {th }}$ percentile male based on height (the $M$ produced by the OpenSim model was found to be independent of subject weight) by scaling the length parameters of the OpenSim upper-limb model using values from [62] (height of 1671 and $1843 \mathrm{~mm}$ for $10^{\text {th }}$ and $90^{\text {th }}$ percentile male, respectively). We were unable to find published measurements of moment arm values for the female upper limb even though moment arms in other body segments have been found to vary significantly between genders $[11,63]$.

Sub-model 3: We previously performed a detailed analysis of the sensitivity of the tremor propagation principles on $I, D$, and $K$ [41]. Entire matrices were changed (scaled by factors from 0.5 to 2), as well as individual matrix entries (including scaling diagonal elements and replacing elements that were initially zero with non-zero values). The individual elements tested were those to which the sub-model was most sensitive to changes. The matrices were scaled together by factors that were deemed conservative, as well as in patterns to mimic physiologicallyplausible conditions such as co-contraction [41]. Because the sensitivity of the full model to the 
impedance matrices is the same as the sensitivity of the third sub-model to these matrices ${ }^{1}$, we did not repeat the sensitivity analysis of $I, D$, and $K$ here but instead relied on the one performed in [41].

\subsubsection{Postures}

Both the inertia matrix and the moment arm matrix depend on the position of the upper limb (see Section 2.2 above). To determine if our conclusions were robust to changes in posture, we repeated our simulations in three additional postures (Figure 2D): with the hand in front of the mouth, representing feeding and grooming activities (posture 2); with the hand in the workspace in front of the abdomen, representing many activities of daily living requiring fine manipulation (posture 3); with the arm somewhat outstretched, representing reaching (posture 4). Joint angles for each posture are given in [41].

${ }^{1}$ To clarify, because the transfer function of the full system is simply the product of the transfer functions of the individual sub-models (see Input-Output Relationships), the magnitude ratio of the full system is simply the product of the magnitude ratios of the individual sub-models: $M=|G|=\left|G_{1} G_{2} G_{3}\right|=\left|G_{1}\right|\left|G_{2}\right|\left|G_{3}\right|=M_{1} M_{2} M_{3}$, where the magnitude ratio of the full model $(M)$ and of the sub-models $\left(M_{1}, M_{2}\right.$, and $\left.M_{3}\right)$ were expressed as the magnitude of the associated transfer function [60]. Since $G_{1}$ and $G_{2}$ are not functions of $I, D$, and $K$, changes in the impedance matrices affect $M_{3}$ but not $M_{1}$ or $M_{2}$. Therefore, changes in $M$ caused by changes in the impedance matrices are the same as changes in $M_{3}$ caused by changes in the impedance matrices. 


\section{RESULTS}

\subsection{Simulations}

The full model transformed the muscle activity into muscle force, joint torque, and finally joint displacement (Figure 3). Both the transient and steady-state responses are clearly observable in the output. Since the system acts as a low-pass filter, higher harmonics are attenuated, and the signal becomes progressively more sinusoidal as it passes from the input (train of triangular pulses) to the output, the steady-state portion of which is practically indistinguishable from a pure sinusoid.

The 105 input-output relationships are characterized by their impulse responses (Figure 4). There is considerable variation in the frequency and settling time of the impulse responses. In particular, the settling times decrease proximal-distally, becoming smallest for responses from distal muscles to distal DOF (Figure 5).

\subsection{Propagation Principles}

The magnitude ratios exhibited resonance below the tremor band (resonance frequencies ranged from 0.02 to $3.2 \mathrm{~Hz}$, mean $1.2 \mathrm{~Hz}$; not shown) but the vast majority (about 95\%) decreased within the tremor band (Figure 6). Although individual magnitude ratios changed significantly in the tremor band, the order of the magnitude ratios (which DOF had the largest magnitude ratio, second-largest magnitude ratio, and so on) was mostly constant throughout the tremor band; the slopes of the various magnitude ratio lines changed together, resulting in 
relatively few crossings, most of which were at the low end of the tremor band (4-6 Hz).

Therefore, tremor propagation patterns (how tremor distributes from input in a given muscle to output in multiple DOF) were quite independent of tremor frequency.

Importantly, all 15 muscles produced the greatest tremor in one of the three most distal DOF (FPS, WFE, or WRD). This was true for the entire tremor band (Figure 6). For most of the tremor band, most of the muscles produced the greatest tremor in WFE ( 7 or 6 muscles, depending on frequency), followed closely by RUD (5 or 6 muscles), and then FPS (3 muscles).

To determine which muscles contribute most to tremor in a given DOF, we investigated the output tremor by DOF, plotting the contribution from each muscle as a phasor (Figure 7). For most of the tremor band, the greatest contributor in DOF 1-7 was: BRA, PECM2, FCU, FCU, BIClong, FCU, and FCU. Thus FCU was the greatest contributor to tremor in 4 of the 7 DOF. Some phasors will add destructively (from Figure 7), but we have insufficient knowledge of the phase of the descending electrical signals to the muscles to conclusively say which phasors will add in this manner (see Section 4.2.4). Note that this is separate from transmission delay; the phasor plots shown assumed the signals were all in phase when leaving the spinal cord.

The pattern of spreading is summarized in Figure 8, which illustrates that (assuming equal inputs in all muscles): 1) tremor increases proximal-distally, and 2) the importance of muscles (to tremor) increases proximal-distally. In particular, most of the tremor appears in the three most distal DOF (FPS, WFE, and WRUD), and most of this tremor comes from: BIC and PT (FPS); FCR, FCU, and ECR (WFE); and FCU, ECR, and ECU (WRUD). 

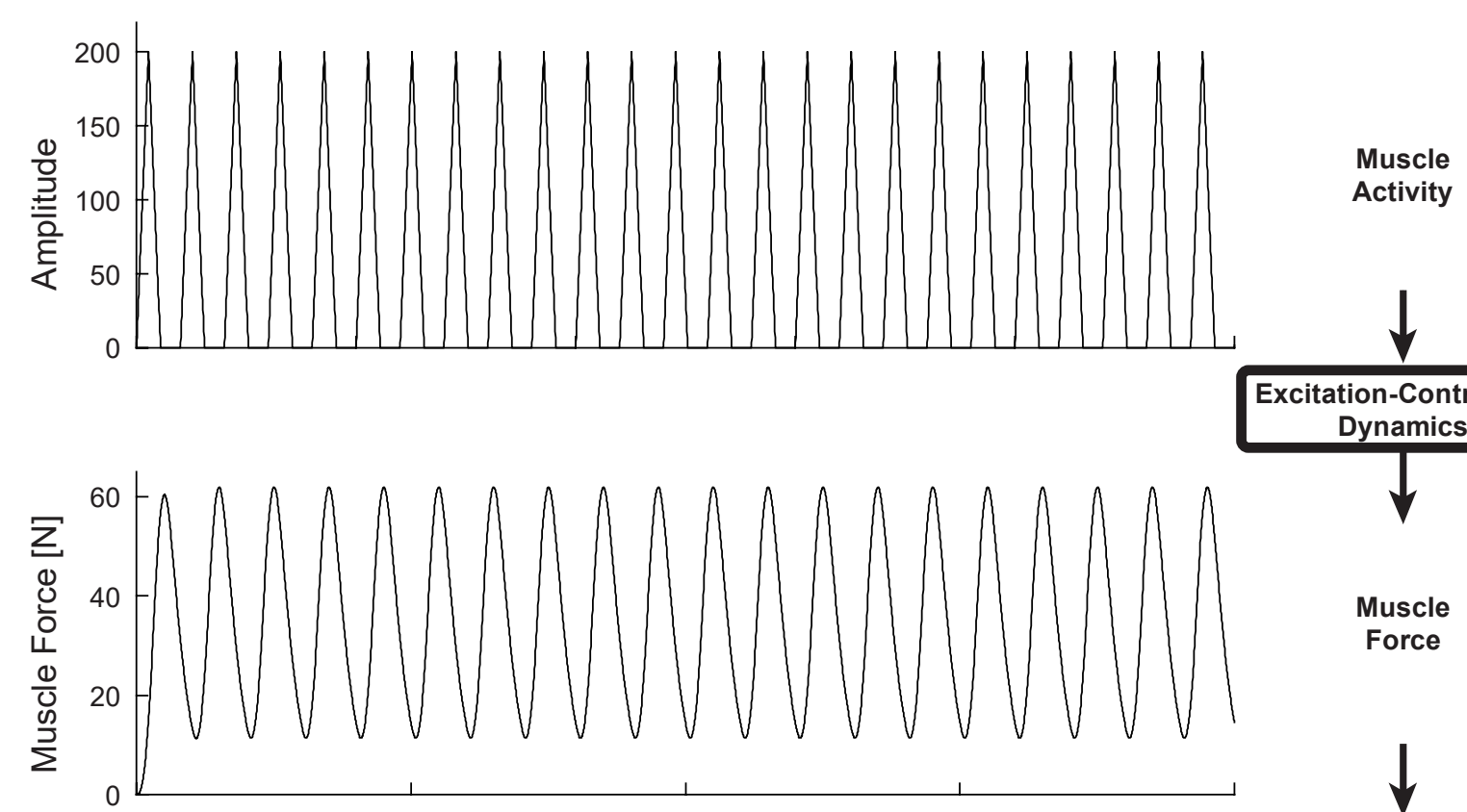

Excitation-Contraction Dynamics
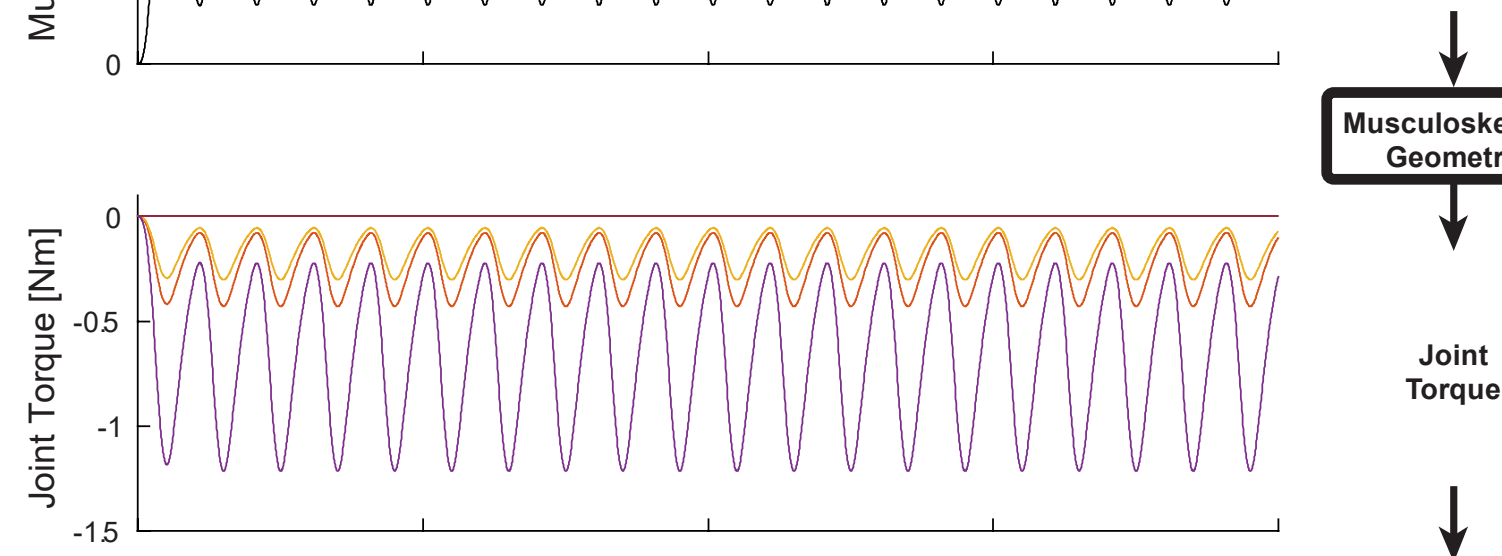

Musculoskeletal Geometry

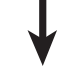

Force

Joint

Torque

Mechanical Impedance

of the Limb

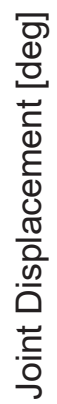
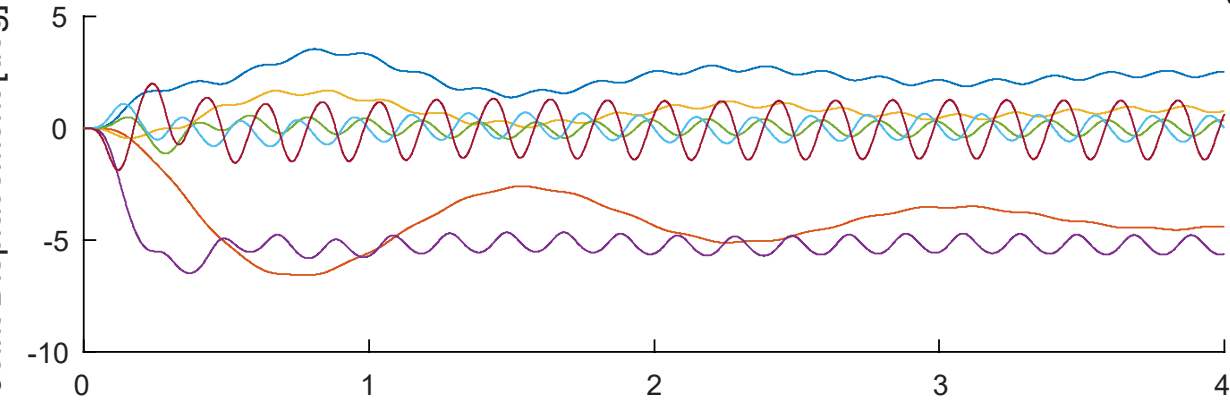

Joint

Displacement

Figure 3. System outputs for an input triangle wave with $110 \mathrm{~ms}$ width at $5 \mathrm{~Hz}$ in TRIlong. The first row shows input muscle activity; the units would be in amplitude, e.g. $\mathrm{mV}$ if sEMG recordings were used as input to the model. The bottom subfigure shows joint displacement from the posture (Posture 1 from [41], shown in Figure 10). This plot includes the transient response, but the rest of the paper focuses only on the steady-state response. 

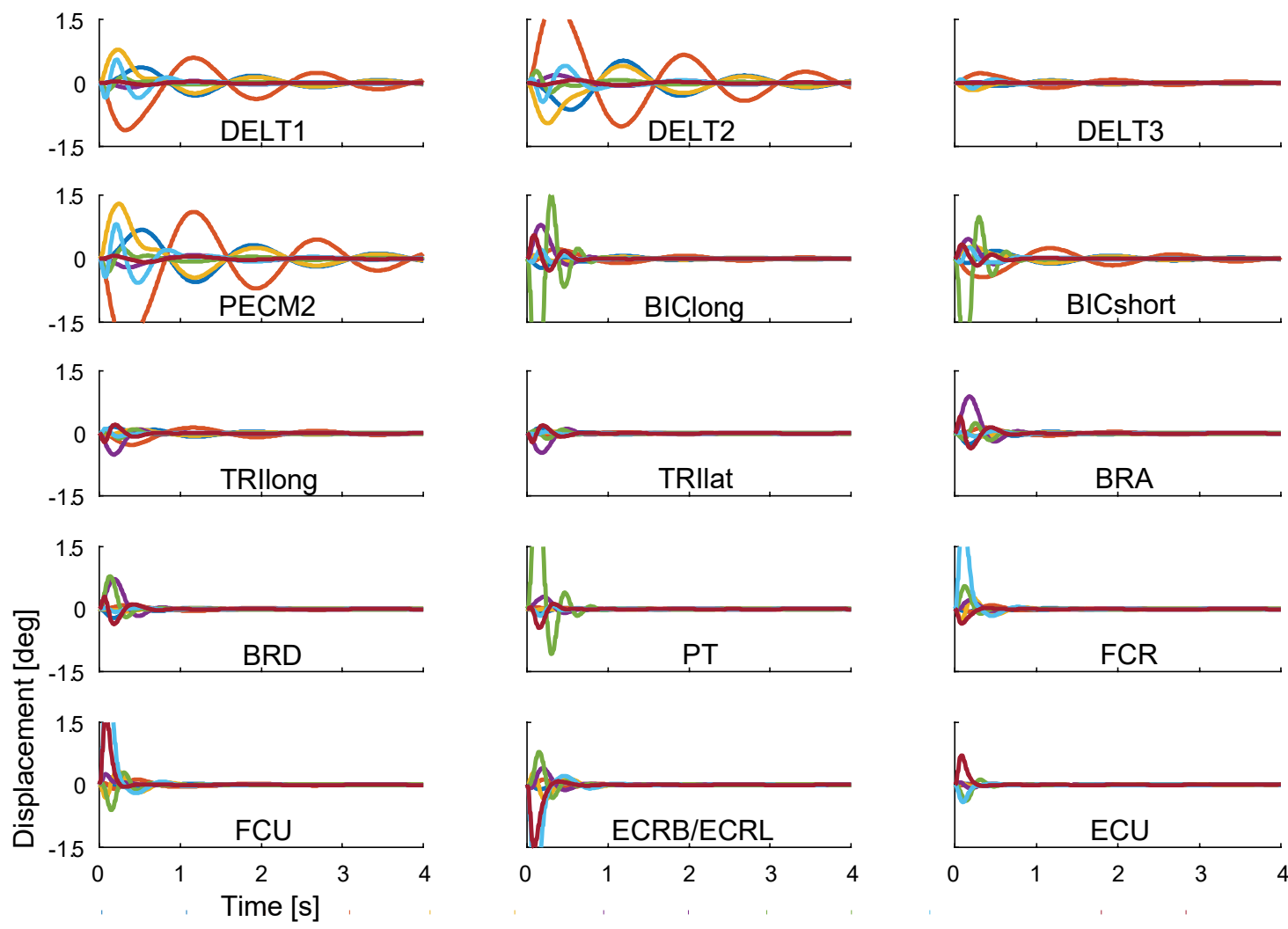

\section{SFE}
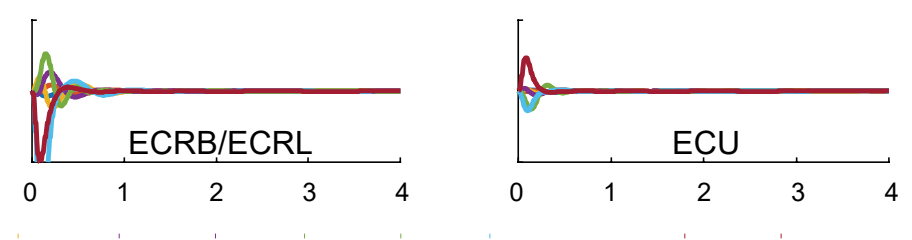

Figure 4. Impulse response from all 15 muscles to all 7 DOF.

To determine how much of the spreading was due to the moment arm matrix $(M)$ vs. inertia $(I)$, we compared the output from the model with default parameter values to a model in which only $M$ contributed to spreading ( $I$ was diagonalized to remove coupling terms) and a model in which only $I$ contributed to spreading ( $M$ was pseudo-diagonalized—see METHODS). For the vast majority of input-output cases, inertia contributed more to spreading than the moment arm matrix (Figure 9; on average, the yellow circles were 1.2X as far from the blue circles as the red circles). The same trends were observed throughout the tremor band. 


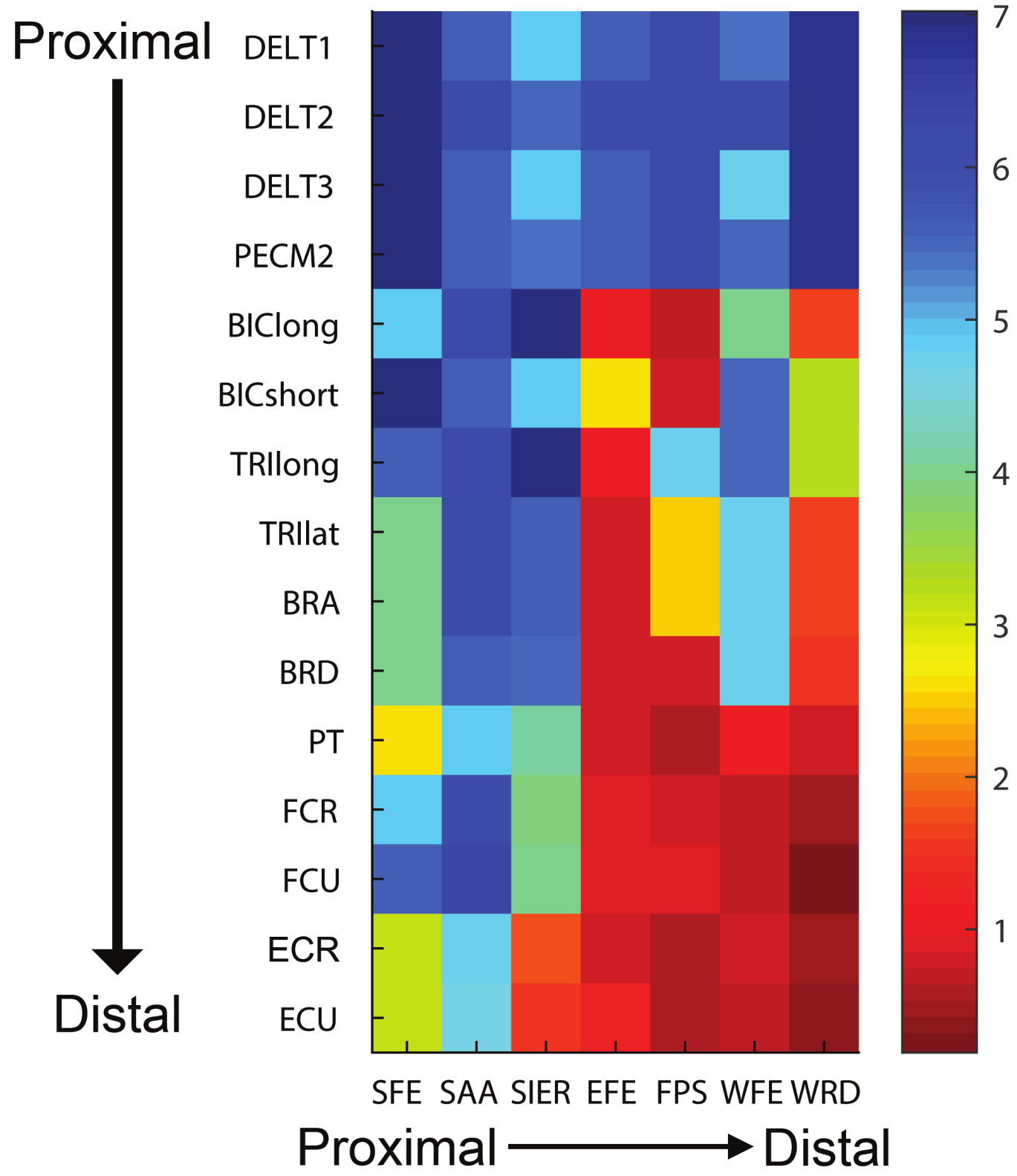

Figure 5. Settling times for transient response. 

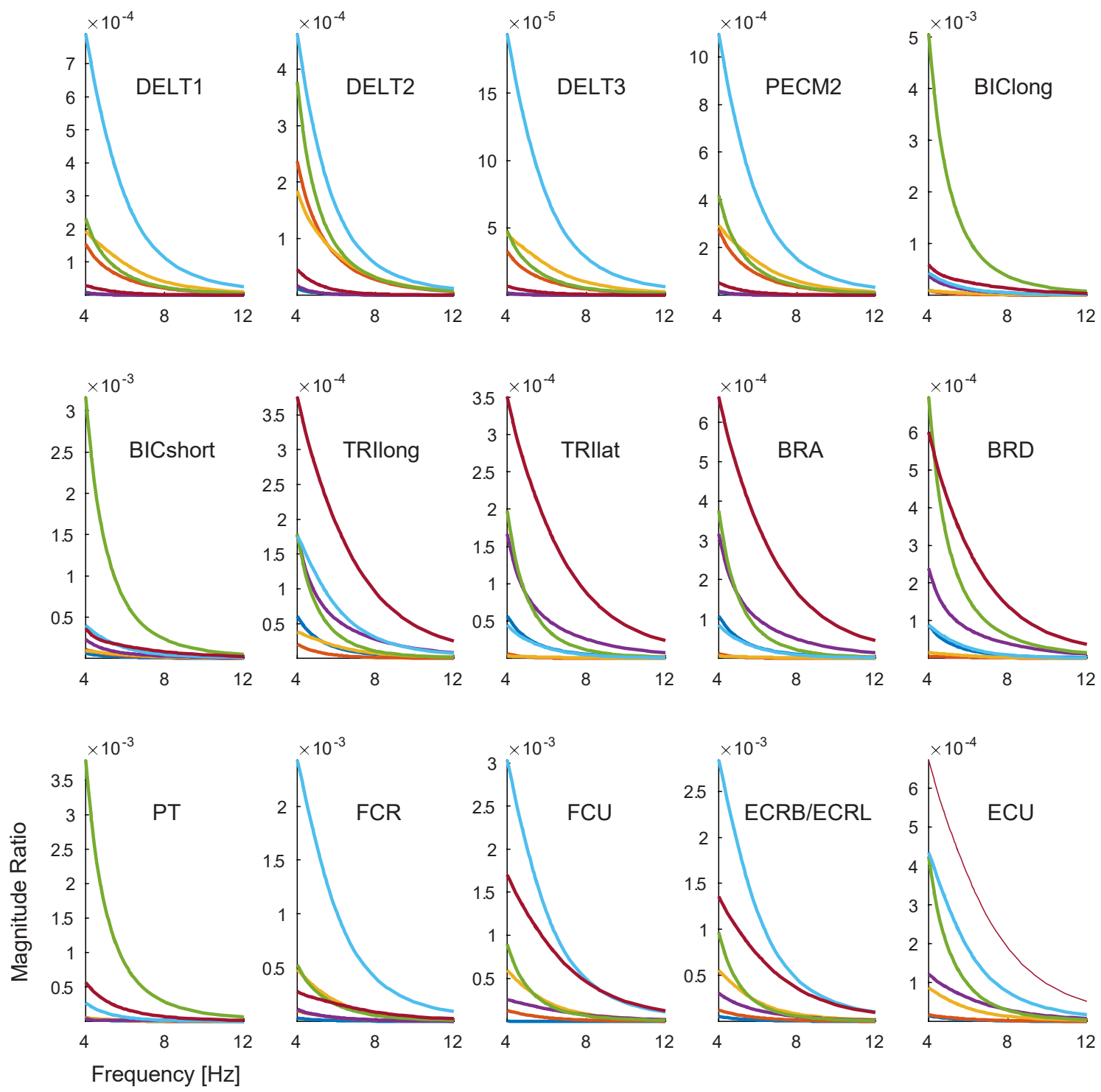

$$
\text { SFE SAA SIER EFE FPS WFE WRD }
$$

Figure 6. Bode plots for input into each muscle and output in all 7 DOF. Shows tremor band, 4$12 \mathrm{~Hz}$. Magnitude is in radians; if input were sEMG, units would be $\mathrm{rad} / \mathrm{mV}$. 


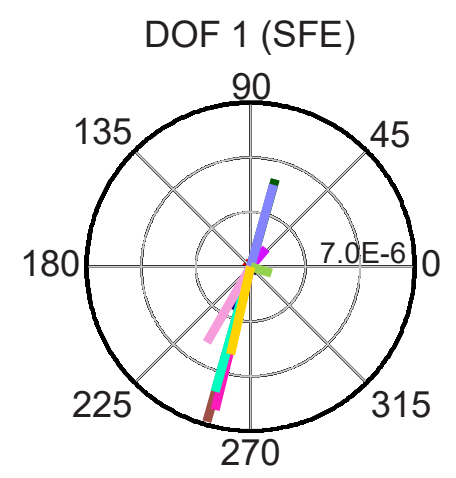

DOF 2 (SAA)
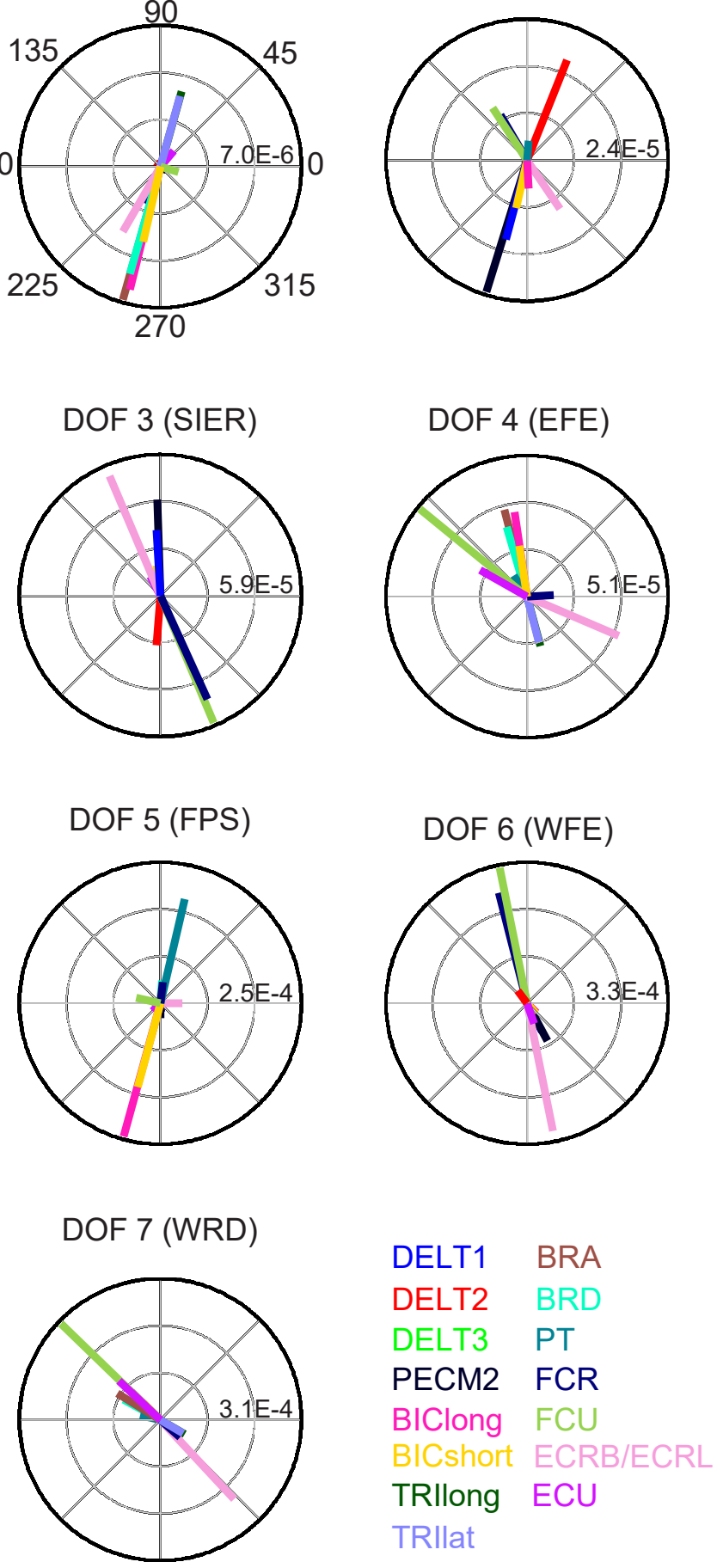

Figure 7. Phasor plots grouped according to output DOF for tremor at $8 \mathrm{~Hz}$. Value on plot gives value at second circle in each plot. 


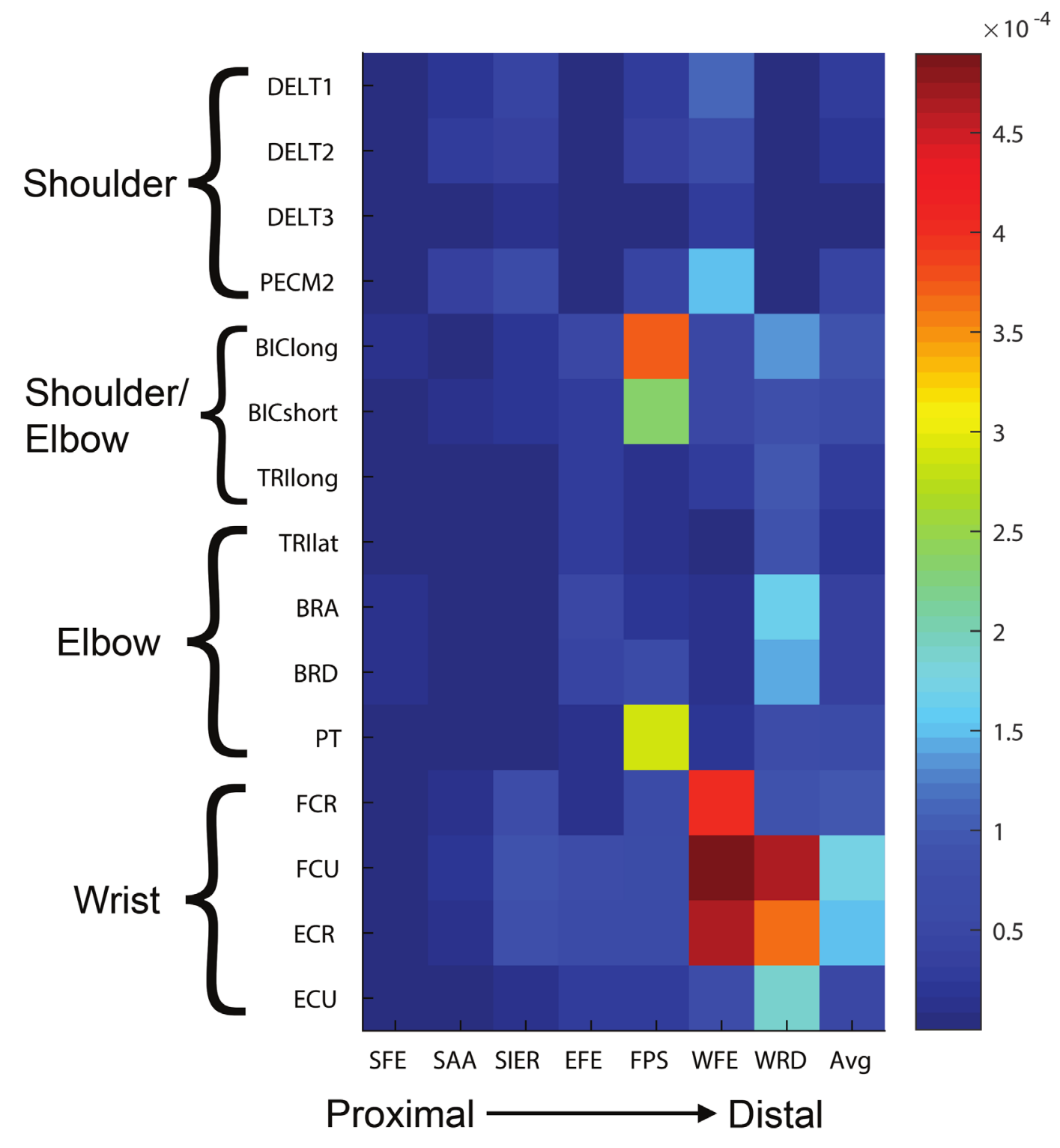

Figure 8. Graphical representation of magnitude ratios for input/output at $8 \mathrm{~Hz}$. Throughout the tremor band, the trends were generally the same. Last column shows mean magnitudes for each row. Magnitude is in radians; if input were sEMG, units would be $\mathrm{rad} / \mathrm{mV}$.

\subsection{Sensitivity Analysis}

As discussed above, we altered parameters within physiological ranges to explore the effects of inaccuracies and inter-subject variability in our model parameters. The goal of the analysis was to determine the robustness of our findings. 

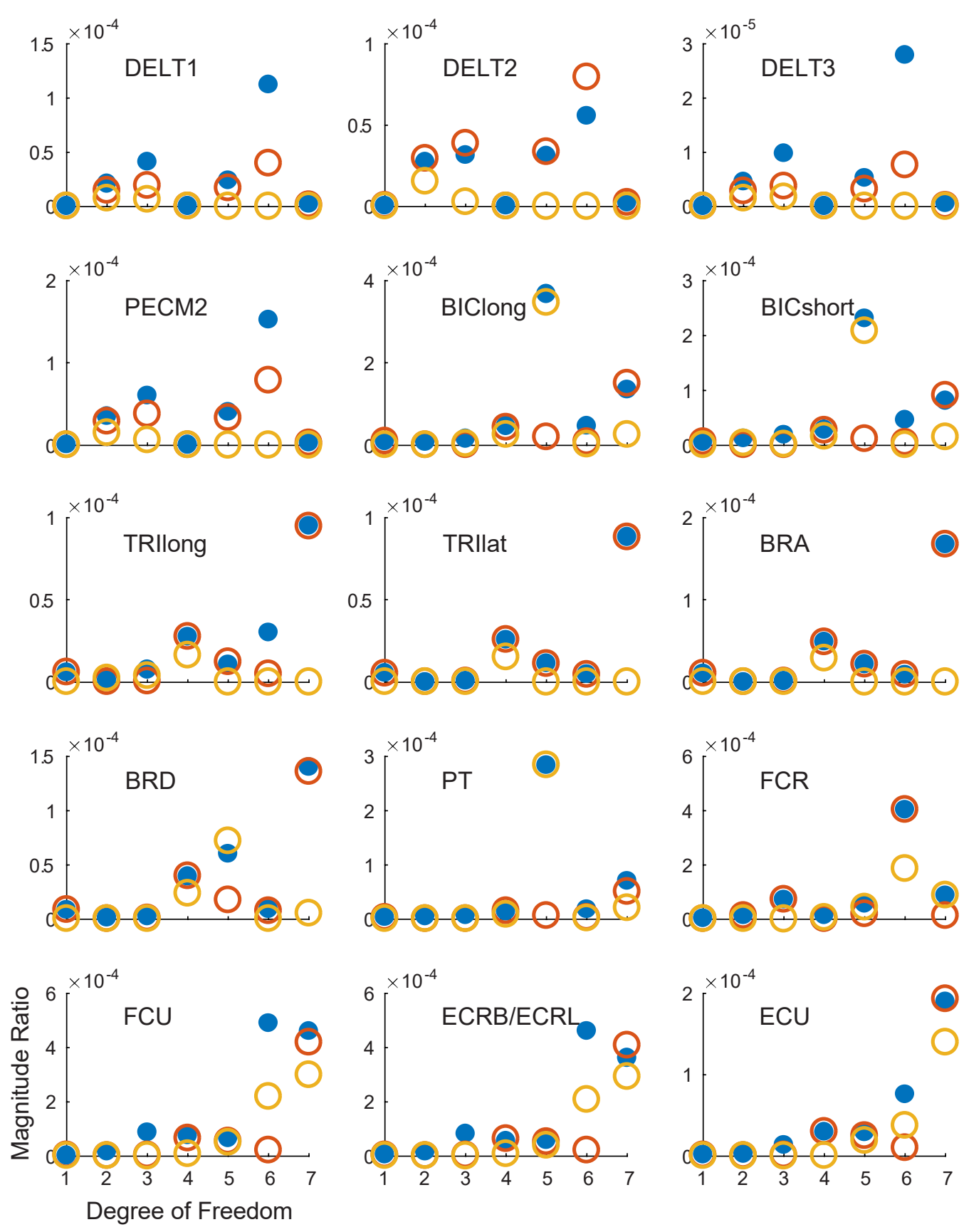

Full Model $\bigcirc$ Due to I Due to $M$

Figure 9. Coupling analysis showing magnitude ratio at $8 \mathrm{~Hz}$ for input into each muscle and output in each DOF (horizontal axis). For the majority of input-output cases, spreading due to $I$ only (red) is more similar to the full model than spreading due to $M$ only (yellow). 


\subsubsection{Input}

The output displacement was quite insensitive to the width of the triangular pulses of the input, even over a large range of widths (50-200 ms); the output was essentially sinusoidal, independent of pulse width. Decreasing the pulse width increased the relative magnitude of the harmonics but, as mentioned above, the harmonics were greatly attenuated by the low-pass filtering properties of the model (Figure 6). By comparison, the magnitude at the fundamental frequency, which was given by the frequency at which the pulses repeated, was relatively unaffected by low-pass filtering.

We also tested different input shapes (a train of narrow rectangular pulses representing impulses, a squared sine wave, and a squared sine wave with multiple frequencies) to test the effect of input shape on the results. The output displacement was found to be quite insensitive to the shape of the input; independent of input shape, the output resembled a pure sinusoid at the fundamental frequency of the input. The low-pass filtering properties of the model attenuated the higher frequency components that distinguish the shapes. Therefore, our results were virtually unaffected by the shape of the input.

\subsubsection{Model Parameters}

Sub-model 1: Increasing the time constants of an overdamped low-pass filter decreases its cut-off frequency, decreasing the magnitude ratio. Therefore, halving and doubling the time constant (default $30 \mathrm{~ms}$ ) simply increased and decreased the magnitude ratio, respectively (Figure 10A). As mentioned above, scaling the value of $C$ associated with a given muscle simply scaled the magnitude ratio of all outputs due to that muscle. 

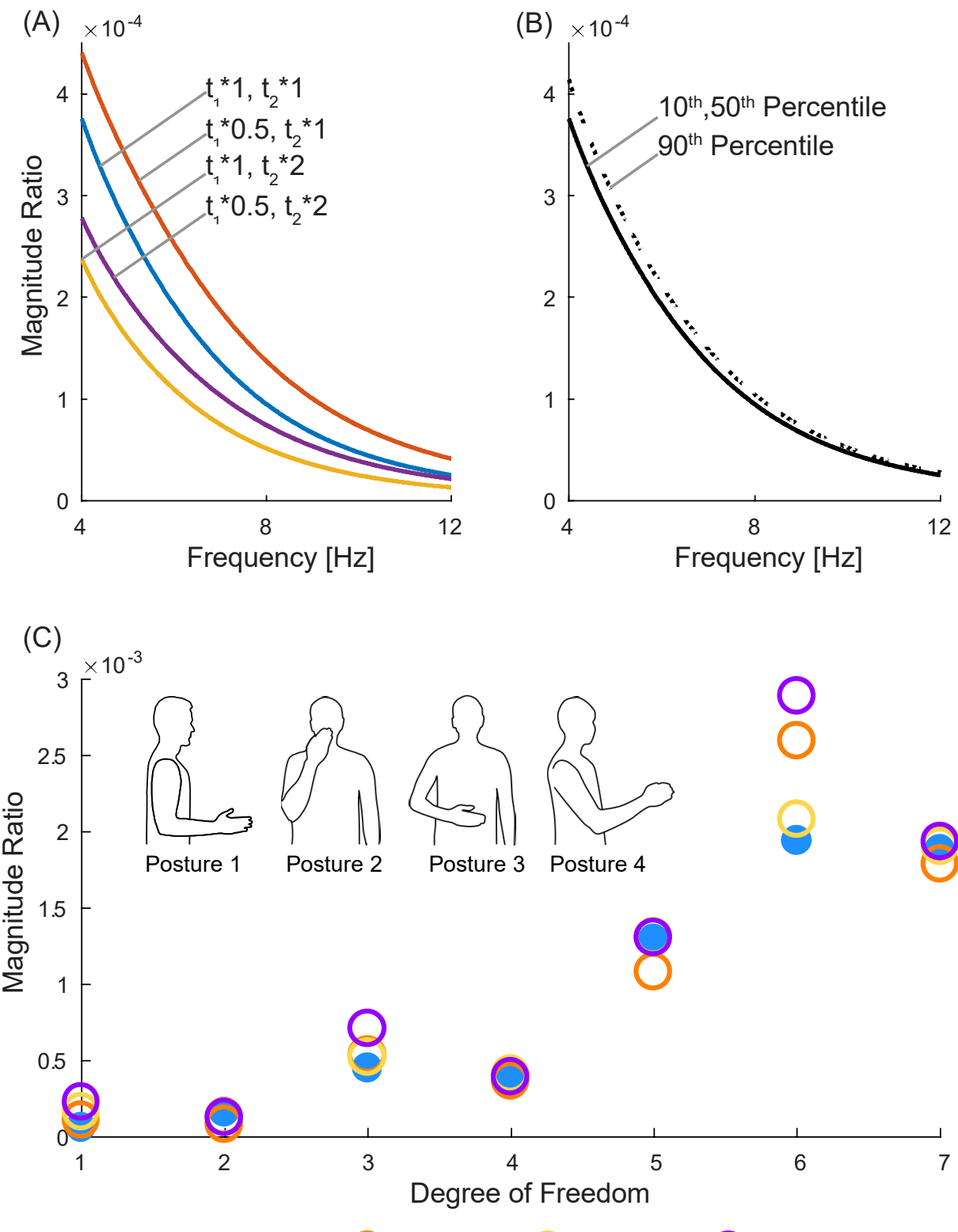

Posture $1 \bigcirc$ Posture $2 \bigcirc$ Posture $3 \bigcirc$ Posture 4

Figure 10. (A) Effect of varying muscle time constants $t_{1}$ and $t_{2}$. (B) Effect of changing moment arms for $10^{\text {th }}$ or $90^{\text {th }}$ percentile male (by height). $10^{\text {th }}$ percentile line is nearly indistinguishable behind $50^{\text {th }}$ percentile line. (C) Changes to total summed magnitude ratio at $8 \mathrm{~Hz}$ for each DOF for different postures. Magnitude is in radians; if input were sEMG, units would be $\mathrm{rad} / \mathrm{mV}$.

Sub-model 2: Changing the OpenSim model from $50^{\text {th }}$ percentile male (default) to $10^{\text {th }}$ and $90^{\text {th }}$ percentile male did not have a large effect on the values of the moment arm matrix, $M$ (Table 2). As expected, default values that were zero did not change, and the vast majority of the 
other default values decreased for the $10^{\text {th }}$ percentile male and increased for the $90^{\text {th }}$ percentile male. Nevertheless, the effect on the magnitude ratio was small (Figure 10B).

Sub-model 3: In a previous sensitivity analysis of the impedance matrices, we found that although physiologically reasonable variations in these matrices can have a significant effect on individual magnitude ratios, "the pattern of propagation remains relatively unchanged" [41]. In summary, Davidson and Charles found the following: increasing $D$ was the only change that always decreased tremor magnitude, increasing stiffness and damping with no change in the damping ratio (similar to co-contraction) usually decreased tremor, and increasing $I$ can decrease or increase tremor [41]. Most importantly, changing the matrices (either by scaling the whole matrix or by scaling individual elements) did not change the patterns of propagation.

\subsubsection{Postures}

To determine the effect of changing the posture of the upper limb, we compared the summed magnitude ratios in four different postures (see METHODS). Changing the posture only changed the $I$ and $M$ matrices, and the effect on the summed magnitude ratios was not large (Figure 10). The most noticeable change occurred in WFE, but the relative magnitudes of tremor in the DOF remained the same; throughout the tremor band, the three most distal DOF still had the greatest tremor amplitude. Creating the Bode plots for the different postures (results not shown) revealed the same trends in terms of tremor spreading to the DOF. For the most part (for 13/15 muscles), a single dominant magnitude ratio was present through the tremor band. The only exception was posture 4 (the most out-stretched position-see Figure 2D), which had significant magnitude ratios in two or more DOF in approximately half of the muscles, though only in the $10-12 \mathrm{~Hz}$ range. 


\section{DISCUSSION}

Our objective was twofold: first, to understand the propagation of tremor; and second, to evaluate the feasibility of determining the origin of tremor. Developing effective tremor suppression devices requires a fundamental understanding of where tremor originates (which muscles), how it propagates through the upper limb, and where it is most severe (which DOF). Both of our objectives aim to guide the development of tremor suppression treatments in the future.

\subsection{Fundamental Principles}

The first and primary goal of this work was to identify principles governing the propagation of tremor from muscle activity to joint displacement. To this end, we 1) determined the extent to which the original tremor propagation principles [41] established for propagation from joint torque to joint displacement (sub-model 3 in Figure 1) held true for propagation from muscle activity to joint displacement (full model in Figure 1), and 2) modified the original principles where necessary to reflect propagation from muscle activity to joint displacement (full model). Thus, the following principles govern simulated tremor propagation from muscle activity to joint displacement.

Principle 1: The distribution of tremor depends strongly on musculoskeletal dynamics. In other words, which DOF has the greatest tremor (output) depends not only on which muscle has 
the most tremorogenic activity (input), but also on how the musculoskeletal system transforms the input into the output (the dynamics of the system). This transformation from tremorogenic muscle activity in multiple muscles to tremulous joint displacement in multiple DOF should be viewed as a multi-input/multi-output process that is not dynamically transparent (i.e. the system does not simply pass inputs straight through to outputs). Rather, the system both low-pass filters and mixes the inputs. More specifically, the excitation-contraction dynamics (sub-model 1) filter, the musculoskeletal geometry (sub-model 2) mixes, and the mechanical impedance of the limb (sub-model 3) filters and mixes.

Principle 2: The spreading of tremor is due to inertial coupling (primarily) and musculoskeletal geometry (secondarily). By comparison, coupling due to joint damping and stiffness played a smaller role [41], and excitation-contraction dynamics played no role. Note that the spreading resulting from inertial coupling not only exceeded the spreading due to musculoskeletal geometry, but was also farther-reaching. To clarify, the moment arm matrix is only capable of spreading tremor from a given muscle to the DOF the muscle crosses, limiting the extent to which the moment arm matrix can spread tremor (e.g. proximal muscles cannot spread to distal DOF, or vice versa). In contrast, the inertia matrix can spread distantly, from torque in proximal DOF to displacement in distal DOF, and vice versa.

Principle 3: Tremor spreads narrowly. Tremorogenic activity in a muscle does not spread significantly to many DOF; instead, most of the tremor caused by a muscle occurs in a small number of DOF. According to our simulations, the frequency response of most muscles was dominated by a single DOF or two DOF (Figure 6). Averaged across the tremor band, the largest magnitude ratio was approximately three times larger than the second-largest ratio. In muscles with two dominant magnitude ratios, these two ratios were approximately three times 
larger than the third-largest ratio. Note that narrow spreading does not imply local spreading; the dominant magnitude ratio was frequently in a DOF that was far from the muscle (e.g. from deltoid muscles to WFE, see Figure 6).

Principle 4: Assuming uniform distribution of tremorogenic activity among upper-limb muscles, tremor increases proximal-distally, and the contribution from muscles increases proximal-distally. In other words, unless proximal muscles receive significantly more tremorogenic muscle activity than distal muscles, most of the tremor will occur in distal DOF, and most of this tremor will come from distal muscles (Figure 8). The proximal-distal increase in tremor was remarkably consistent: throughout the entire tremor band, all 15 muscles produced the greatest tremor in one of the three most distal DOF. This increase was not due to excitationcontraction dynamics: muscle time constants were assumed equal for all muscles, and peak force (represented in the gain matrix $C$ ) roughly decreases from proximal to distal (Table 1), which would produce the opposite effect. Nor does the proximal-distal increase in tremor come from musculoskeletal geometry, which lacks any clear proximal-distal trend in moment arms (Table 2). Instead, the proximal-distal increase in tremor is "caused by proximal-distal differences in impedance. Going from proximal to distal, inertia decreases more rapidly than stiffness... This creates a proximal-distal increase in the natural frequency, which pushes the resonance band to higher frequencies, elevating the magnitude ratios in the tremor band" [41].

The original tremor propagation principles [41] included two additional principles that depend only on sub-model 3. These principles are unchanged by the addition of sub-models 1 and 2 (see Section 2.7.2), so we repeat them here for completeness: Increasing inertia can decrease or increase tremor (Principle 5) and increasing viscoelasticity can decrease or increase tremor (Principle 6). 


\subsection{Robustness of Principles}

Here we discuss the results of the sensitivity analyses in the context of the fundamental principles. Our tests simulated within plausible ranges for inaccuracy or inter-subject variability. Generally, the principles were quite robust to changes to the input, model parameters, and posture within these ranges.

\subsubsection{Input}

Because the full system is a low-pass filter with low cut-off frequency (Figure 6), harmonics are strongly attenuated, and the final output of the system is almost identical to a pure sine at the fundamental frequency of the input pulse train, no matter the shape of the pulses. The fundamental frequency of the input pulse train lies in the 4-12 Hz tremor band. Consequently, the steady-state output of the system is fully characterized (except for a scaling factor) by the frequency response of the system in the tremor band. Since the magnitude of the input to individual muscles varies by subject, we formulated the tremor propagation principles on the assumption of equal inputs into all muscles. Therefore, the principles depend entirely on the system, and not on the input.

\subsubsection{Model Parameters}

As mentioned above, sub-model 1 (representing excitation-contraction dynamics) lowpass filters the input but does not mix inputs. The cut-off frequency of sub-model 1 is given by the muscle time constants. In the model with default parameters, the cut-off frequency was below the tremor band (around $3 \mathrm{~Hz}$ ). Although the principles are based on the fact that the harmonics are filtered out (see above), the exact value of the cut-off frequency is not important for two reasons: 1) as long as the cut-off frequency is below the frequency of the first harmonic (8-24 
$\mathrm{Hz}$, depending on the fundamental frequency), the harmonics are attenuated relative to the fundamental frequency, and 2) since sub-model 3 also low-pass filters with a low cut-off frequency (range of 1 to $6 \mathrm{~Hz}$, mean $3 \mathrm{~Hz}$ ), the harmonics would be attenuated even if the cut-off frequency of sub-model 1 were much higher.

Likewise, the exact values of the gain matrix $C$ (representing peak force in each muscle) were not critical to the principles. Scaling $C$ uniformly has no impact whatsoever on the principles. Scaling $C$ non-uniformly would change the contribution of each muscle to the total output (essentially scaling all phasors of the same color in Figure 7 by the same amount), but the principles are quite robust to physiologically reasonable scaling; Principle 1 is not directly influenced by $C$, and although it may be possible to construct a $C$ that would invalidate Principle 2 , it would require just the right set of values in $C$, which would be highly unlikely. No scaling of $C$ will invalidate Principle 3 because the outputs of a given muscle are always scaled the same.

The first part of Principle 4 (tremor increases proximal-distally) is insensitive to nonuniform scaling. Some muscles have a proximal DOF in their top three DOF, and increasing the values of $C$ for these muscles (and not the others) will increase tremor in the proximal DOF relative to the distal DOF. For example, if the peak forces of muscles 1-4 were scaled by a factor of 10, one of the proximal DOF would enter the top three. However, for every muscle, the top DOF is a distal one, so the most tremor will always be in a distal DOF, no matter what value is used for $C$. The second part of Principle 4 (the contribution from muscles increases proximaldistally) does depend on $C$. Increasing the proximal values of $C$ would increase the contribution of the proximal muscles to tremor. However, one would have to increase the proximal values of $C$ by approximately $2 \mathrm{X}$ before the proximal muscles would provide the same contribution to 
tremor as the distal muscles. Lastly, because $C$ represents peak muscle force, it could be adjusted to better reflect individual subjects if subject-specific estimates of peak force were available.

Changing the moment arm matrix over a large range $\left(10^{\text {th }}\right.$ to $90^{\text {th }}$ percentile male $)$ did not affect the principles. No moment-arm values that were zero became non-zero, or vice versa, so basic coupling patterns remained unchanged. In addition, the observed change in moment-arm values was only on the order of $10 \%$ and scaled more or less in unison. Consequently, tremor propagation patterns were minimally affected, including which DOF exhibited the greatest tremor: the three distal DOF dominated throughout the tremor band in all three models $\left(10^{\text {th }}\right.$, $50^{\text {th }}$, and $90^{\text {th }}$ percentile).

As discussed above, Davidson and Charles thoroughly explored the effects of changing the $I, D$, and $K$ matrices [41]. In summary, they found that the principles were quite insensitive to physiologically reasonable changes in the impedance matrices; although changes sometimes produced large changes in the frequency response of the system, the pattern of propagation remained relatively unchanged (see [41] for details).

\subsubsection{Postures}

Changing posture did not have a large effect on the magnitude ratio (Figure 10), so it is clear that Principle 1 was unaffected. Repeating the analysis shown in Figure 9 for the three additional postures (results not shown) confirmed that Principle 2 was not affected by changes in posture. Principle 3 was robust to posture changes in most cases: averaged across all postures, 13 of the 15 muscles exhibited a single dominant magnitude ratio. If a muscle had a second magnitude ratio similar to the largest one, the others were comparatively small. Finally, the three distal DOF dominated throughout the tremor band (Principle 4) in all four postures. 


\subsubsection{Phase}

When dealing with periodic inputs and outputs, the phase is crucial to understanding how the signals combine and, in this case, how tremor will affect the resultant kinematics. Our knowledge, however, about the phase shift between outputs is limited. To clarify, the phase shift between outputs depends on the phase shift between inputs and the phase shift due to the system's dynamics. The phase shift between tremorogenic activity exciting the spinal cord (phase shift between inputs) is unknown, however, even for antagonist muscles [64]. A number of studies have also suggested that feedback contributes significantly to tremor (e.g. [30, 65]). A conservative estimate of the additional phase shift in the inputs due to transmission delay determined that its effects would be small (30 degrees or less; see below). This situation is similar to how the magnitude of the outputs from the system are unknown because the magnitude of the inputs are unknown. The same approach applies: we can assume equality among the inputs and draw out fundamental principles that are based on that assumption.

Fortunately, more is known about the phase shift due to the system. As shown in Figure 7 (which assumed equal phase in all muscles), tremor due to antagonist muscles is often almost exactly out of phase, including dominant phasors. This would lead to destructive interference; however, vector summing (results not shown) determined that if inputs to all muscles were assumed to be in phase, the greatest tremor was still in one of the three distal DOF (Principle 4), though occasionally the order of DOF was different. For the other principles, it is possible to change the phase shifts of the inputs in such a way as to cancel out any inputs, so it is possible to violate the principles, but this would be highly unlikely in reality. In summary, because the principles were based on individual phasors and not the vector sum, they are quite robust to changes in phase shifts. 
It is important to consider the potential effects of transmission delay. For example, it is theoretically possible that WFE will end up having very little tremor because the phasors cancel each other out (even though individually they are quite large). While phase delay would not affect the length of individual phasors, it could rotate some phasors relative to others so they would sum differently.

A conservative estimate indicated that phase delay could be 30 degrees at most, i.e. the phasor from a proximal (e.g. shoulder) muscle (in Figure 7) could be rotated $30^{\circ}$ relative to a distal (e.g. wrist) muscle. This estimate assumed the worst-case, i.e. a signal travelling slowly $(80 \mathrm{~m} / \mathrm{s})$ the farthest distance (from shoulder to wrist). Based on this conservative estimate, we do not believe the principles would change if the model included transmission delay. Rotating distal phasors by $30^{\circ}$ relative to proximal phasors is not enough to change the addition from constructive to destructive (or even to neutral); that requires a change of $180^{\circ}\left(90^{\circ}\right.$ for neutral). Only phasors from muscles that are significantly removed from each other may have significant transmission delay relative to each other. However, in Figure 7 the dominant phasors (in a given DOF) often belong to muscles that are close to each other, so they would not have significant transmission delay between them.

\subsection{Limitations}

As this is the first investigation of tremor propagation from muscle to joint displacement throughout the upper limb (from shoulder to wrist), we deliberately used a simple (linear, timeinvariant) model to focus on first-order, steady-state effects. Consequently, our model ignored transient responses and higher-order effects, including non-linear dynamics and time-varying impedance parameters. That said, postural tremor involves relatively small displacements from an equilibrium position, so non-linear dynamics and time-varying changes in moment arms and 
inertia are expected to be small. Furthermore, there is no reason to expect systematic variations in muscle time constants or joint viscoelasticity while holding a posture for a 30 s period. We therefore expect the principles established above to be robust to non-linear dynamics and timevarying changes in impedance parameters associated with postural tremor. Nevertheless, our conclusions cannot be extrapolated to tremor during voluntary movement (kinetic tremor) or even tremor in a postural task requiring modulation of joint torque or co-contraction (e.g. in response to unstable perturbations such as during tool use).

Numerous studies have suggested that afferent feedback could play a significant role in tremor (e.g. $[30,65,66])$. Such feedback could cause tremor to spread to other DOF through "neural coupling" even if they are not mechanically coupled. Here we have focused on propagation through mechanical coupling and have excluded neural coupling for the sake of tractability, but future work should include the effect of neural coupling on tremor propagation.

The range of motion tested by the four postures is relatively small. We would expect large changes in postures to result in larger changes in tremor propagation, especially at the limits of the upper limb's range of motion (e.g. limb fully extended).

Lastly, these principles were based entirely on simulation results and were not validated by experiments with actual subjects. Our research group is currently compiling a dataset for ET patients that would include both the input (recorded sEMG for all 15 muscles) and the output (joint kinematics for the 7 DOF). In our future work, we will input real data into our simple model and compare the results with real outputs. This could confirm whether this simplified model is sufficient to capture the fundamental principles of tremor propagation. 


\subsection{Conclusion}

The aim of this research was to establish the principles of tremor propagation from tremorogenic muscle activity to hand displacement. As discussed in Davidson and Charles [41], tremor propagation through the upper limb should be viewed as the result of a multi-input/ multioutput system with dynamics. These system dynamics include filtering due to excitationcontraction dynamics and the mechanical impedance of the limb. All of the original principles from [41] for the mechanical impedance sub-model were found to be true for the entire model, and some of the principles were expanded. In summary, we accomplished our objective by determining the following principles: 1) The distribution of tremor depends strongly on musculoskeletal dynamics; 2) The spreading of tremor is due to inertial coupling (primarily) and musculoskeletal geometry (secondarily); 3) Tremor spreads narrowly; 4) Assuming uniform distribution of tremorogenic activity among upper-limb muscles, tremor increases proximaldistally, and the contribution from muscles increases proximal-distally.

We anticipate that these principles will add to the body of knowledge in the field of tremor and guide future research. This should enable the development of tremor treatments that suppress tremor in a more informed and effective manner. For example, [67] explored treatment that targets specific muscles based on biomechanical patterns (sensors captured movement in various upper limb DOF, then injections were made based on which muscles contribute to tremor in that DOF). This sort of treatment approach requires an understanding of how tremor from the different muscles manifests in the DOF of the upper limb.

Future work will include modeling reflex activity and comparing our model to real inputoutput data, with the ultimate goal of developing the method and increasing our understanding sufficiently so that we can extract the origin and propagation of tremor. Our end goal is to have 
the following occur: 1) a patient with tremor walks into a specialized clinic and is instrumented with motion capture and/or EMG sensors (or marker-less motion capture is performed); 2) the data is passed into a decomposition algorithm; 3) we are able to tell the patient that their tremor is mostly due to tremorogenic muscle activity in specific muscles; 4) clinicians present a customized treatment plan and/or suppression device. This would allow the tremor patient to regain normal function and perform the activities of daily life. 


\section{REFERENCES}

[1] K. P. Bhatia, P. Bain, N. Bajaj, R. J. Elble, M. Hallett, E. D. Louis, et al., "Consensus Statement on the classification of tremors. from the task force on tremor of the International Parkinson and Movement Disorder Society," Movement Disorders, vol. 33, pp. 75-87, 2018.

[2] A. Anouti and W. C. Koller, "Tremor disorders. Diagnosis and management," Western Journal of Medicine, vol. 162, pp. 510-513, 1995.

[3] J. Á. Gallego, E. Rocon, J. M. Belda-Lois, and J. L. Pons, "A neuroprosthesis for tremor management through the control of muscle co-contraction," Journal of NeuroEngineering and Rehabilitation, vol. 10, pp. 36-36, 2013.

[4] G. Deuschl, P. Bain, and M. Brin, "Consensus Statement of the Movement Disorder Society on Tremor," Movement Disorders, vol. 13, pp. 2-23, 1998.

[5] E. D. Louis and J. J. Ferreira, "How common is the most common adult movement disorder? Update on the worldwide prevalence of essential tremor," Movement Disorders, vol. 25, pp. 534-541, 2010.

[6] E. D. Louis and R. Ottman, "How Many People in the USA Have Essential Tremor? Deriving a Population Estimate Based on Epidemiological Data," vol. 4, ed, 2014.

[7] W. Koller, N. Biary, and S. Cone, "Disability in essential tremor: effect of treatment," Neurology, vol. 36, pp. 1001-1004, 1986.

[8] A. I. Tröster, R. Pahwa, J. A. Fields, C. M. Tanner, and K. E. Lyons, "Quality of life in Essential Tremor Questionnaire (QUEST): Development and initial validation," Parkinsonism \& Related Disorders, vol. 11, pp. 367-373, 2005. 
[9] E. D. Louis, B. Rohl, and C. Rice, "Defining the Treatment Gap: What Essential Tremor Patients Want That They Are Not Getting," vol. 5, ed, 2015.

[10] R. E. T. A. Zesiewicz E. D. Louis and et al., "Practice Parameter: Therapies for essential tremor," Neurology, vol. 64, pp. 2008-2020, 2005.

[11] G. Deuschl, J. Raethjen, H. Hellriegel, and R. Elble, "Treatment of patients with essential tremor," The Lancet Neurology, vol. 10, pp. 148-161, 2011.

[12] E. D. Louis, E. Rios, and C. Henchcliffe, "How are We Doing With the Treatment of Essential Tremor (ET)? Persistence of ET Patients on Medication: Data from 528 Patients in Three Settings," vol. 17, ed, 2010, pp. 882-884.

[13] N. L. Diaz and E. D. Louis, "Survey of medication usage patterns among essential tremor patients: Movement disorder specialists vs. general neurologists," Parkinsonism \& Related Disorders, vol. 16, pp. 604-607, 2010.

[14] S. A. Schneider and G. Deuschl, "The Treatment of Tremor," Neurotherapeutics, vol. 11, pp. 128-138, 2014.

[15] A. Chopra, B. T. Klassen, and M. Stead, "Current clinical application of deep-brain stimulation for essential tremor," Neuropsychiatric Disease and Treatment, vol. 9, pp. 1859-1865, 2013.

[16] E. D. Flora, C. L. Perera, A. L. Cameron, and G. J. Maddern, "Deep brain stimulation for essential tremor: A systematic review," Movement Disorders, vol. 25, pp. 1550-1559, 2010.

[17] R. E. Wharen, M. S. Okun, B. L. Guthrie, R. J. Uitti, P. Larson, K. Foote, et al., "Thalamic DBS with a constant-current device in essential tremor: A controlled clinical trial," Parkinsonism \& Related Disorders, 2017.

[18] T. A. Dembek, M. T. Barbe, M. Åström, M. Hoevels, V. Visser-Vandewalle, G. R. Fink, et al., "Probabilistic mapping of deep brain stimulation effects in essential tremor," NeuroImage: Clinical, vol. 13, pp. 164-173, 2017.

[19] R. Pahwa, K. E. Lyons, S. B. Wilkinson, R. K. Simpson Jr, W. G. Ondo, D. Tarsy, et al., "Long-term evaluation of deep brain stimulation of the thalamus," Journal of Neurosurgery, vol. 104, pp. 506-512, 2006. 
[20] A. Ramirez-Zamora, H. Boggs, and J. G. Pilitsis, "Reduction in DBS frequency improves balance difficulties after thalamic DBS for essential tremor," Journal of the Neurological Sciences, vol. 367, pp. 122-127, 2016.

[21] P. Blomstedt and M. I. Hariz, "Are complications less common in deep brain stimulation than in ablative procedures for movement disorders?," Stereotactic and Functional Neurosurgery, vol. 84, pp. 72-81, 2006.

[22] L. C. Shih, K. LaFaver, C. Lim, E. Papavassiliou, and D. Tarsy, "Loss of benefit in VIM thalamic deep brain stimulation (DBS) for essential tremor (ET): How prevalent is it?," Parkinsonism \& Related Disorders, vol. 19, pp. 676-679, 2013.

[23] O. Sydow, S. Thobois, F. Alesch, and J. D. Speelman, "Multicentre European study of thalamic stimulation in essential tremor: a six year follow up," Journal of neurology, neurosurgery, and psychiatry, vol. 74, pp. 1387-1391, 2003.

[24] J. F. Baizabal-Carvallo, M. N. Kagnoff, J. Jimenez-Shahed, R. Fekete, and J. Jankovic, "The safety and efficacy of thalamic deep brain stimulation in essential tremor: 10 years and beyond," Journal of Neurology, Neurosurgery \& Psychiatry, vol. 85, pp. 567-572, 2014.

[25] M. Kestenbaum, B. Ford, and E. D. Louis, "Estimating the Proportion of Essential Tremor and Parkinson's Disease Patients Undergoing Deep Brain Stimulation Surgery: Five-Year Data From Columbia University Medical Center (2009-2014)," Movement Disorders Clinical Practice, vol. 2, pp. 384-387, 2015.

[26] S. Maxwell, "A modulated-energy-dissipation manipulator and application to suppressing human arm tremor," 1990.

[27] J. Kotovsky and M. J. Rosen, "A wearable tremor-suppression orthosis," Journal of rehabilitation research and development, vol. 35, pp. 373-387, 1998.

[28] M. M. Camut, R. Eduardo, P. Jose, B. Juan Manuel, and Stephane, "Evaluation of a wearable orthosis and an associated algorithm for tremor suppression," Physiological Measurement, vol. 28, pp. 415-415, 2007.

[29] E. Rocon, J. M. Belda-Lois, A. F. Ruiz, M. Manto, J. C. Moreno, and J. L. Pons, "Design and validation of a rehabilitation robotic exoskeleton for tremor assessment and suppression," IEEE transactions on neural systems and rehabilitation engineering : a 
publication of the IEEE Engineering in Medicine and Biology Society, vol. 15, pp. 367378, 2007.

[30] T. C. Britton, P. D. Thompson, B. L. Day, J. C. Rothwell, L. J. Findley, and C. D. Marsden, "Rapid wrist movements in patients with essential tremor. The critical role of the second agonist burst," Brain : a journal of neurology, vol. 117 ( Pt 1, pp. 39-47, 1994.

[31] M. Lauk, B. Köster, J. Timmer, B. Guschlbauer, G. Deuschl, and C. H. Lücking, "Sideto-side correlation of muscle activity in physiological and pathological human tremors," Clinical Neurophysiology, vol. 110, pp. 1774-1783, 1999.

[32] M. Lauk, J. Timmer, B. Guschlbauer, B. Hellwig, and C.-H. Lücking, "Variability of frequency and phase between antagonistic muscle pairs in pathological human tremors," Muscle \& Nerve, vol. 24, pp. 1365-1370, 2001.

[33] B. Koster, G. Deuschl, M. Lauk, J. Timmer, B. Guschlbauer, and C. Lucking, "Essential tremor and cerebellar dysfunction: abnormal ballistic movements," Journal of Neurology, Neurosurgery, and Psychiatry, vol. 73, pp. 400-405, 2002.

[34] P. Trillenberg, J. Fuhrer, A. Sprenger, A. Hagenow, D. Kompf, R. Wenzelburger, et al., "Eye-hand coordination in essential tremor," Movement disorders : official journal of the Movement Disorder Society, vol. 21, pp. 373-379, 2006.

[35] H. Wang, Q. Yu, M. M. Kurtis, A. G. Floyd, W. A. Smith, and S. L. Pullman, "Spiral analysis-improved clinical utility with center detection," Journal of neuroscience methods, vol. 171, pp. 264-270, 2008.

[36] D. A. Heldman, J. Jankovic, D. E. Vaillancourt, J. Prodoehl, R. J. Elble, and J. P. Giuffrida, "Essential tremor quantification during activities of daily living," Parkinsonism \& related disorders, vol. 17, pp. 537-542, 2011.

[37] C. L. Pulliam, S. R. Eichenseer, C. G. Goetz, O. Waln, C. B. Hunter, J. Jankovic, et al., "Continuous in-home monitoring of essential tremor," Parkinsonism and Related Disorders, vol. 20, pp. 37-40, 2014.

[38] L. Avanzino, M. Bove, A. Tacchino, P. Ruggeri, Giannini, Alessandro, et al., "Cerebellar involvement in timing accuracy of rhythmic finger movements in essential tremor," European Journal of Neuroscience, vol. 30, pp. 1971-1979, 2009. 
[39] G. M. Earhart, M. Hong, S. D. Tabbal, and J. S. Perlmutter, "Effects of thalamic stimulation frequency on intention and postural tremor," Experimental neurology, vol. 208, pp. 257-263, 2007.

[40] J. Herzog, W. Hamel, R. Wenzelburger, M. Pötter, M. O. Pinsker, J. Bartussek, et al., "Kinematic analysis of thalamic versus subthalamic neurostimulation in postural and intention tremor," Brain, vol. 130, pp. 1608 LP-1625, 2007.

[41] A. D. Davidson and S. K. Charles, "Fundamental Principles of Tremor Propagation in the Upper Limb," Annals of Biomedical Engineering, vol. 45, pp. 1133-1147, 2017.

[42] D. A. Winter, Biomechanics and Motor Control of Human Movement: John Wiley \& Sons, Inc., 2009.

[43] E. Burdet, Human robotics: The MIT Press, Cambridge, Massachusetts, 2013.

[44] M. Haruno and D. M. Wolpert, "Optimal control of redundant muscles in step-tracking wrist movements," Journal of Neurophysiology, vol. 94, pp. 4244-4255, 2005.

[45] K. R. S. Holzbaur, W. M. Murray, G. E. Gold, and S. L. Delp, "Upper limb muscle volumes in adult subjects," Journal of Biomechanics, vol. 40, pp. 742-749, 2007.

[46] K. R. Holzbaur, S. L. Delp, G. E. Gold, and W. M. Murray, "Moment-generating capacity of upper limb muscles in healthy adults," J Biomech, vol. 40, pp. 2442-2449, 2007.

[47] K. R. Saul, X. Hu, C. M. Goehler, M. E. Vidt, M. Daly, A. Velisar, et al., "Benchmarking of dynamic simulation predictions in two software platforms using an upper limb musculoskeletal model," Comput Methods Biomech Biomed Engin, vol. 18, pp. 1445$1458,2015$.

[48] K. R. S. Holzbaur, W. M. Murray, and S. L. Delp, "A Model of the Upper Extremity for Simulating Musculoskeletal Surgery and Analyzing Neuromuscular Control," Annals of Biomedical Engineering, vol. 33, pp. 829-840, 2005.

[49] G. Wu, F. C. T. van der Helm, H. E. J. Veeger, M. Makhsous, P. Van Roy, C. Anglin, et al., "ISB recommendation on definitions of joint coordinate systems of various joints for the reporting of human joint motion-Part II: shoulder, elbow, wrist and hand," Journal of Biomechanics, vol. 38, pp. 981-992, 2005. 
[50] D. Formica, S. K. Charles, L. Zollo, E. Guglielmelli, N. Hogan, and H. I. Krebs, "The passive stiffness of the wrist and forearm," Journal of Neurophysiology, vol. 108, pp. 1158-1166, 2012.

[51] W. B. Drake and S. K. Charles, "Passive Stiffness of Coupled Wrist and Forearm Rotations," Annals of Biomedical Engineering, vol. 42, pp. 1853-1866, 2014.

[52] A. L. Pando, H. Lee, W. B. Drake, N. Hogan, and S. K. Charles, "Position-dependent characterization of passive wrist stiffness," IEEE transactions on bio-medical engineering, vol. 61, pp. 2235-2244, 2014.

[53] P. de Leva, "Adjustments to Zatsiorsky-Seluyanov's segment inertia parameters," Journal of Biomechanics, vol. 29, pp. 1223-1230, 1996.

[54] P. Corke, Robotics, Vision and Control: Fundamental Algorithms In MATLAB ${ }^{\circledR}$ Second, Completely Revised, Extended And Updated Edition: Springer International Publishing, 2017.

[55] Y. Matsumoto, M. Seki, T. Ando, Y. Kobayashi, H. Iijima, M. Nagaoka, et al., "Analysis of EMG signals of patients with essential tremor focusing on the change of tremor frequency," Conference proceedings : ... Annual International Conference of the IEEE Engineering in Medicine and Biology Society. IEEE Engineering in Medicine and Biology Society. Annual Conference, vol. 2012, pp. 2244-2250, 2012.

[56] F. He, P. G. Sarrigiannis, S. A. Billings, H. Wei, J. Rowe, C. Romanowski, et al., "Nonlinear interactions in the thalamocortical loop in essential tremor: A model-based frequency domain analysis," Neuroscience, vol. 324, pp. 377-389, 2016.

[57] G. Grimaldi and M. Manto, "Tremor: From Pathogenesis to Treatment," Synthesis Lectures on Biomedical Engineering, vol. 3, pp. 1-212, 2008.

[58] R. Nisticò, D. Pirritano, M. Salsone, F. Novellino, F. Del Giudice, M. Morelli, et al., "Synchronous pattern distinguishes resting tremor associated with essential tremor from rest tremor of Parkinson's disease," Parkinsonism \& Related Disorders, vol. 17, pp. 30$33,2011 / 01 / 01 / 2011$.

[59] I. Milanov, "Electromyographic differentiation of tremors," Clinical Neurophysiology, vol. 112, pp. 1626-1632. 
[60] W. J. Palm, System dynamics, 3rd ed.: McGraw-Hill, New York, NY, 2014.

[61] Y. Matsumoto, M. Seki, Y. Nakashima, T. Ando, Y. Kobayashi, H. Iijima, et al., "Algorithm to demodulate an electromyogram signal modulated by essential tremor," ROBOMECH Journal, vol. 4, p. 15, 2017/05/31 2017.

[62] M. Parkinson. (2014, August 22). Anthropometric Data Explorer. Available: http://tools.openlab.psu.edu/tools/explorer.php

[63] P. J. Moga, M. Erig, D. B. Chaffin, and M. A. Nussbaum, "Torso muscle moment arms at intervertebral levels T10 through L5 from CT scans on eleven male and eight female subjects," Spine (Phila Pa 1976), vol. 18, pp. 2305-9, Nov 1993.

[64] J. A. Gallego, J. L. Dideriksen, A. Holobar, J. Ibanez, V. Glaser, J. P. Romero, et al., "The phase difference between neural drives to antagonist muscles in essential tremor is associated with the relative strength of supraspinal and afferent input," $J$ Neurosci, vol. 35, pp. 8925-37, Jun 102015.

[65] D. J. Pedrosa, E.-L. Quatuor, C. Reck, K. A. M. Pauls, C. A. Huber, V. VisserVandewalle, et al., "Thalamomuscular Coherence in Essential Tremor: Hen or Egg in the Emergence of Tremor?," The Journal of Neuroscience, vol. 34, p. 14475, 2014.

[66] J. L. Dideriksen, R. M. Enoka, and D. Farina, "A Model of the Surface Electromyogram in Pathological Tremor," IEEE Transactions on Biomedical Engineering, vol. 58, pp. 2178-2185, 2011.

[67] O. Samotus, F. Rahimi, J. Lee, and M. Jog, "Functional Ability Improved in Essential Tremor by IncobotulinumtoxinA Injections Using Kinematically Determined Biomechanical Patterns - A New Future," PLOS ONE, vol. 11, p. e0153739, 2016.

[68] H. Baruh, "Applied Dynamics," ed: CRC Press, 2014.

[69] M. A. Sherman, A. Seth, and S. L. Delp, "What is a Moment Arm? Calculating Muscle Effectiveness in Biomechanical Models Using Generalized Coordinates," in Proceedings of the ASME 2013 International Design Engineering Technical Conferences and Computers and Information in Engineering Conference, Portland, Oregon, USA, 2013.

[70] O. Bock and N. Wenderoth, "Dependence of peripheral tremor on mechanical perturbations: a modeling study," Biological Cybernetics, vol. 80, pp. 103-108, 1999. 
[71] R. B. Stein and M. N. Oguztoreli, "Tremor and other oscillations in neuromuscular systems," Biological cybernetics, vol. 22, pp. 147-157, 1976.

[72] E. Pierrot-Deseilligny and D. Burke, THE CIRCUITRY OF THE HUMAN SPINAL CORD: ITS ROLE IN MOTOR CONTROL AND MOVEMENT DISORDERS. Cambridge: Cambridge University Press, 2005.

[73] V. M. McClelland, S. Miller, and J. A. Eyre, "Short latency heteronymous excitatory and inhibitory reflexes between antagonist and heteronymous muscles of the human shoulder and upper limb," Brain Research, vol. 899, pp. 82-93, 2001/04/27/ 2001.

[74] J. B. J. Smeets and J. J. D. van der Gon, "An unsupervised neural network model for the development of reflex co-ordination," Biological Cybernetics, vol. 70, pp. 417-425, 1994/03/01 1994.

[75] T. MIYASAKA;, Y.-J. SUN;, A. NAITO;, and M. Shindo, "INHIBITORY PROJECTIONS FROM THE BICEPS BRACHII TO THE PRONATOR TERES MOTONEURONS IN THE HUMAN " Neuroscience Research, vol. 25, p. S183, 1996

[76] A. Créange, M. Faist, R. Katz, and A. Pénicaud, "Distribution of heteronymous Ia facilitation and recurrent inhibition in the human deltoid motor nucleus," Experimental Brain Research, vol. 90, pp. 620-624, 1992/09/01 1992.

[77] T. Miyasaka, A. Naito, H. Morita, Y.-J. Sun, M. Chishima, and M. Shindo, "Inhibitory neural connection from the brachioradialis to the pronator teres in human," Neuroscience Research, vol. 31, p. S159, 1998/01/01/ 1998.

[78] S. Kobayashi, M. Hayashi, K. Shinozaki, M. Nito, W. Hashizume, T. Miyasaka, et al., "Oligosynaptic inhibition of group I afferents between the brachioradialis and flexor carpi radialis in humans," Neuroscience Research, vol. 110, pp. 37-42, 2016/09/01/ 2016.

[79] K. Shinozaki, M. Nito, S. Kobayashi, M. Hayashi, T. Miyasaka, W. Hashizume, et al., "Monosynaptic facilitation of group I afferents between brachioradialis and extensor carpi radialis in humans," Neuroscience Research, vol. 114, pp. 30-34, 2017/01/01/ 2017.

[80] A. Naito, M. Shindo, T. Miyasaka, Y. J. Sun, H. Momoi, and M. Chishima, "Inhibitory projections from pronator teres to biceps brachii motoneurones in human," Exp Brain Res, vol. 121, pp. 99-102, Jul 1998. 
[81] T. Miyasaka, A. Naito, M. Shindo, S. Kobayashi, and K. Shinozaki, "Inhibitory and excitatory projections from the median nerve (Med) to brachioradialis (BR) motoneurones (MN) in humans," Neuroscience Research, vol. 39, pp. PE1-147, 2001.

[82] C. D. Manning and P. Bawa, "Heteronymous reflex connections in human upper limb muscles in response to stretch of forearm muscles," Journal of Neurophysiology, vol. 106, p. 1489, 2011.

[83] G. R. Chalmers and P. Bawa, "Synaptic connections from large afferents of wrist flexor and extensor muscles to synergistic motoneurones in man," Experimental Brain Research, vol. 116, pp. 351-358, 1997/09/01 1997.

[84] S. C. Elliott, J. R. Hanson, J. Wellington, and C. M. Alexander, "Reflex control of posterior shoulder muscles from arm afferents in healthy people," Journal of Electromyography and Kinesiology, vol. 21, pp. 1087-1091, 2011/12/01/2011. 


\section{APPENDIX A. Moment Arm Conversion}

Here we will outline how to convert moment arms between different Euler angle sequences. We will follow an example converting from a $Y X Y$ (or 2-1-2) order to a $Z X Y$ (or 3-12) Euler angle sequence.

\section{Euler Angle Sequences}

Let Frame $A$ be an inertial base frame, with Frames $A$ ' and $A$ "' as intermediate frames; the resultant, rotated frame is $B$. The first rotation occurs about one of the $A$ axes; the second rotation occurs about one of the $A$ ' axes; the third rotation occurs about one of the $A$ "' axes. The three rotation angles are called Euler angles and can be grouped into a single array, $\vec{E}=[\psi \theta \varphi]^{T}$. In this example, the Euler angle order is $Z X Y$, meaning rotate $\psi$ about $Z_{A}, \theta$ about $X_{A}$, and $\varphi$ about $Y_{A}{ }^{\prime \prime}$. It is important to note some of the axes will be the same, i.e. $Z_{A}=Z_{A}$, $X_{A}{ }^{\prime}=X_{A}{ }^{\prime \prime}$, and $Y_{A}{ }^{\prime \prime}=$ $Y_{B}$

\section{Rotation Matrices}

Each of the transformations, from one frame to the next in the sequence, can be represented by a rotation matrix, $R$. Here we will use the convention used in Robotics, e.g. in [54], (as opposed to the convention used in Dynamics, e.g. [68]): 


$$
\left[\begin{array}{l}
X_{A} \\
Y_{A} \\
Z_{A}
\end{array}\right]=R\left[\begin{array}{l}
X_{B} \\
Y_{B} \\
Z_{B}
\end{array}\right]
$$

Where each of the three steps of the Euler angle sequence makes one part of the rotation matrix: $R=R_{Z_{A} \psi} R_{X_{A^{\prime}} \theta} R_{Y_{A^{\prime \prime}}}$.

\section{Angular Velocities}

The angular velocity about the fixed (inertial or "base") frame, $\vec{\omega}=\left[\omega_{1} \omega_{2} \omega_{3}\right]^{T}=\left[\omega_{X A}\right.$ $\left.\omega_{Y A} \omega_{Z A}\right]^{T}$, is related to the Euler angle rates $\dot{\vec{E}}=[\dot{\psi} \dot{\theta} \dot{\varphi}]^{T}$ via a matrix which we will call $C$ for this example, i.e. $\vec{\omega}=C \dot{\vec{E}}_{Z X Y}$. This is often referred to, e.g., in [68], as "the $B$ matrix", i.e. $\dot{\vec{\omega}}=$ $B \dot{\vec{E}}$. Please note that this matrix has nothing to do with our final frame, $B$. Continuing the $Z X Y$ example, $\dot{\psi}$ is about $Z_{A}$, so it will contribute:

$$
\left[\begin{array}{l}
\omega_{1} \\
\omega_{2} \\
\omega_{3}
\end{array}\right]=\left[\begin{array}{l}
0 \\
0 \\
\dot{\psi}
\end{array}\right]
$$

$\dot{\theta}$ is about $X_{A}$, so it will contribute:

$$
\left[\begin{array}{l}
\omega_{1} \\
\omega_{2} \\
\omega_{3}
\end{array}\right]=R_{Z \psi}\left[\begin{array}{l}
\dot{\theta} \\
0 \\
0
\end{array}\right]
$$

because $R_{Z \psi}$ is the transformation that rotates the axes to the $X_{A}$ ' frame. In the same way, $\dot{\varphi}$ is about $Y_{A}$,", so it will contribute:

$$
\left[\begin{array}{l}
\omega_{1} \\
\omega_{2} \\
\omega_{3}
\end{array}\right]=R_{Z \psi} R_{X \theta}\left[\begin{array}{l}
0 \\
\dot{\phi} \\
0
\end{array}\right]
$$

because $R_{Z \psi}$ and $R_{X \theta}$ are the transformations that take us from the base frame, $A$, to the $Y_{A}$, frame. This results in: 


$$
\left[\begin{array}{c}
\omega_{1} \\
\omega_{2} \\
\omega_{3}
\end{array}\right]=\left[\begin{array}{l}
0 \\
0 \\
\dot{\psi}
\end{array}\right]+R_{Z \psi}\left[\begin{array}{l}
\dot{\theta} \\
0 \\
0
\end{array}\right]+R_{Z \psi} R_{X \theta}\left[\begin{array}{c}
0 \\
\dot{\phi} \\
0
\end{array}\right]
$$

When arranged in matrix form, this gives:

$$
\left[\begin{array}{c}
\omega_{1} \\
\omega_{2} \\
\omega_{3}
\end{array}\right]=\left[\begin{array}{ccc}
0 & \cos (\psi) & -\sin (\psi) \cos (\theta) \\
0 & \sin (\psi) & \cos (\psi) \cos (\theta) \\
1 & 0 & \sin (\theta)
\end{array}\right]\left[\begin{array}{c}
\dot{\psi} \\
\dot{\theta} \\
\dot{\phi}
\end{array}\right]=C\left[\begin{array}{c}
\dot{\psi} \\
\dot{\theta} \\
\dot{\phi}
\end{array}\right]
$$

Or $\vec{\omega}=C \dot{\vec{E}}_{Z X Y}$. Suppose $Z X Y$ is the Euler angle sequence we want for our angular velocities or torques. Now suppose the same procedure is followed for a different Euler angle sequence - a sequence that we have information in but we want to convert our angular velocities out of this sequence. In this example, we will use $Y X Y$, i.e. $\vec{E}_{Y X Y}=[\alpha \beta \gamma]^{T}$ where we rotate $\alpha$ about $Y_{A}, \beta$ about $X_{A}$, and $\gamma$ about $Y_{A^{\prime}}$ (where these are different $X_{A}$, and $Y_{A^{\prime \prime}}$ axes than in $Z X Y$ ). We find:

$$
\left[\begin{array}{c}
\omega_{1} \\
\omega_{2} \\
\omega_{3}
\end{array}\right]=\left[\begin{array}{c}
0 \\
\dot{\alpha} \\
0
\end{array}\right]+R_{Y \alpha}\left[\begin{array}{c}
\dot{\beta} \\
0 \\
0
\end{array}\right]+R_{Y \alpha} R_{X \beta}\left[\begin{array}{l}
0 \\
\dot{\gamma} \\
0
\end{array}\right]
$$

Resulting in a matrix $D$ :

$$
\begin{aligned}
& {\left[\begin{array}{l}
\omega_{1} \\
\omega_{2} \\
\omega_{3}
\end{array}\right]=\left[\begin{array}{ccc}
0 & \cos (\alpha) & \sin (\alpha) \sin (\beta) \\
1 & 0 & \cos (\beta) \\
0 & -\sin (\alpha) & \cos (\alpha) \sin (\beta)
\end{array}\right]\left[\begin{array}{l}
\dot{\alpha} \\
\dot{\beta} \\
\dot{\gamma}
\end{array}\right]=D\left[\begin{array}{c}
\dot{\alpha} \\
\dot{\beta} \\
\dot{\gamma}
\end{array}\right]} \\
& \text { Where } \vec{\omega}=D \dot{\vec{E}}_{Y X Y} .
\end{aligned}
$$

\section{Conversion}

We can now convert $\dot{\vec{E}}_{Z X Y}$ to $\dot{\vec{E}}_{Y X Y}$ by finding the equivalent $\vec{\omega}$, or the equivalent vectors in the base frame, $A$. Since, by Equations A.6 and A. 8 we have: 


$$
\left[\begin{array}{c}
\omega_{1} \\
\omega_{2} \\
\omega_{3}
\end{array}\right]=C\left[\begin{array}{c}
\dot{\psi} \\
\dot{\theta} \\
\dot{\phi}
\end{array}\right]=D\left[\begin{array}{c}
\dot{\alpha} \\
\dot{\beta} \\
\dot{\gamma}
\end{array}\right]
$$

Or $C \dot{\vec{E}}_{Z X Y}=D \dot{\vec{E}}_{Y X Y}$. We can find $\dot{\vec{E}}_{Z X Y}$ given $\dot{\vec{E}}_{Y X Y}$ easily with $\dot{\vec{E}}_{Z X Y}=C^{-1} D \dot{\vec{E}}_{Y X Y}$ because the $C$ and $D$ matrices are $3 \times 3$ and are invertible in most cases. For this example, $C$ is invertible if $\cos \theta \neq 0$ and $D$ is invertible if $\sin \beta \neq 0$.

\section{Converting Torques}

OpenSim, which was used to calculate the moment arm matrix, $M$, follows the ISB convention [49], which is a $Y X Y$ Euler angle sequence [47] for angles at the shoulder. Since angular velocities and torques are often defined in the same way (as vectors about axes in intermediate frames), the same process can be undertaken to convert torques from one Euler angle sequence to another: $\vec{\tau}_{Z X Y}=C^{-1} D \vec{\tau}_{Y X Y}$. For convenience, we will define $E=C^{-1} D$. Therefore,

$$
\vec{\tau}_{Z X Y}=E \vec{\tau}_{Y X Y}
$$

\section{Moment Arms}

OpenSim defines the moment arm matrix, $M$, as $M=\frac{\tau}{f}$. $\tau$ is "a scalar representing the effective torque acting about a joint angle $[q]$ that is due to the scalar tension force $[f]$ generated by muscle activation" (definitions are quoted from [69] with notation adjusted). The values used in OpenSim for $M$ are derived in [69] as $M=\frac{d l}{d q}$, where $l=l(q)$ is "the musculotendon path length along which the [force $f$ ] is acting" [69]. This illustrates why the Euler angle sequence is so crucial. The moment arms were calculated about angles, $q$, and using $l=l(q)$ that are 
dependent on how the angles are described. Using a different Euler angle sequence would mean the axes used were different, resulting in different $q$ and $l$, and a different $M$.

\section{Converting Moment Arms}

From OpenSim we can obtain $M_{Y X Y}$, but for our model (or any system in another Euler angle sequence) we require $M$ to be transformed to our Euler angle sequence, here $M_{Z X Y}$. We

know that a given set of forces, $\vec{f}$, will result in different torques in the two systems: $\vec{f}=$ $M_{Y X Y}{ }^{-1} \vec{\tau}_{Y X Y}=M_{Z X Y}{ }^{-1} \vec{\tau}_{Z X Y}$. Substituting in Equation A.10:

$$
\begin{aligned}
& M_{Y X Y}{ }^{-1} \vec{\tau}_{Y X Y}=M_{Z X Y}{ }^{-1} E \vec{\tau}_{Y X Y} \\
& M_{Y X Y}{ }^{-1}=M_{Z X Y}{ }^{-1} E \\
& M_{Y X Y}{ }^{-1} E^{-1}=M_{Z X Y}{ }^{-1}
\end{aligned}
$$

Taking the inverse of each side we obtain $M_{Z X Y}=E M_{Y X Y}$. This same procedure can be applied to convert the moment arms matrix from OpenSim, $M_{Y X Y}$, to another Euler angle sequence. Note that in our model this conversion applied only to the first three rows of $M$ (entries for the shoulder DOF). 


\section{APPENDIX B. Feedback}

In addition to expanding the upper-limb tremor dynamics model to include muscle activity, this work also laid the foundation for future research concerning closed-loop feedback. Modeling reflexes is anything but trivial, so we followed a methodical approach in which we systematically addressed multiple feedback parameters. These parameters included saturation, homonymous vs. heteronymous feedback, and delay. Homonymous feedback is defined as from a given muscle to the spinal cord back to the same muscle; heteronymous feedback is from a given muscle to the spinal cord, and then to a different muscle.

We simplified the analysis by adding the feedback at the level of DOF instead of muscles. While not as accurate as modeling feedback at the muscle level, this approach captures the main effects of feedback (since muscle force due to feedback combines as torque at the DOFlevel) and greatly simplifies the simulation.

Feedback was successfully simulated using a simple model adapted by Bock and Wenderoth [70] from Stein and Oguztoreli [71] (Figure 1). According to this model, "proprioceptive feedback is modeled by a proportional and a differential component, where the latter is passed through a saturation non-linearity and both through a delay element" [70]. While from literature it is simple to identify useful saturation nonlinearity functions, the output of these functions depends entirely on the magnitude of the input. 
Our models were robust during these preliminary tests. Our review of literature did not find any values for the saturation limits, so we performed a series of tests to see how the system was affected by different saturation levels. The saturation block was placed on the derivative portion of the feedback loop [70]. We concluded that application of a saturation limit was unnecessary for our model if we can assume that the saturation limit was low and we only analyzed situations when the output is stable without saturation. Our work on delay was still in its earliest stages, so it is not discussed here.

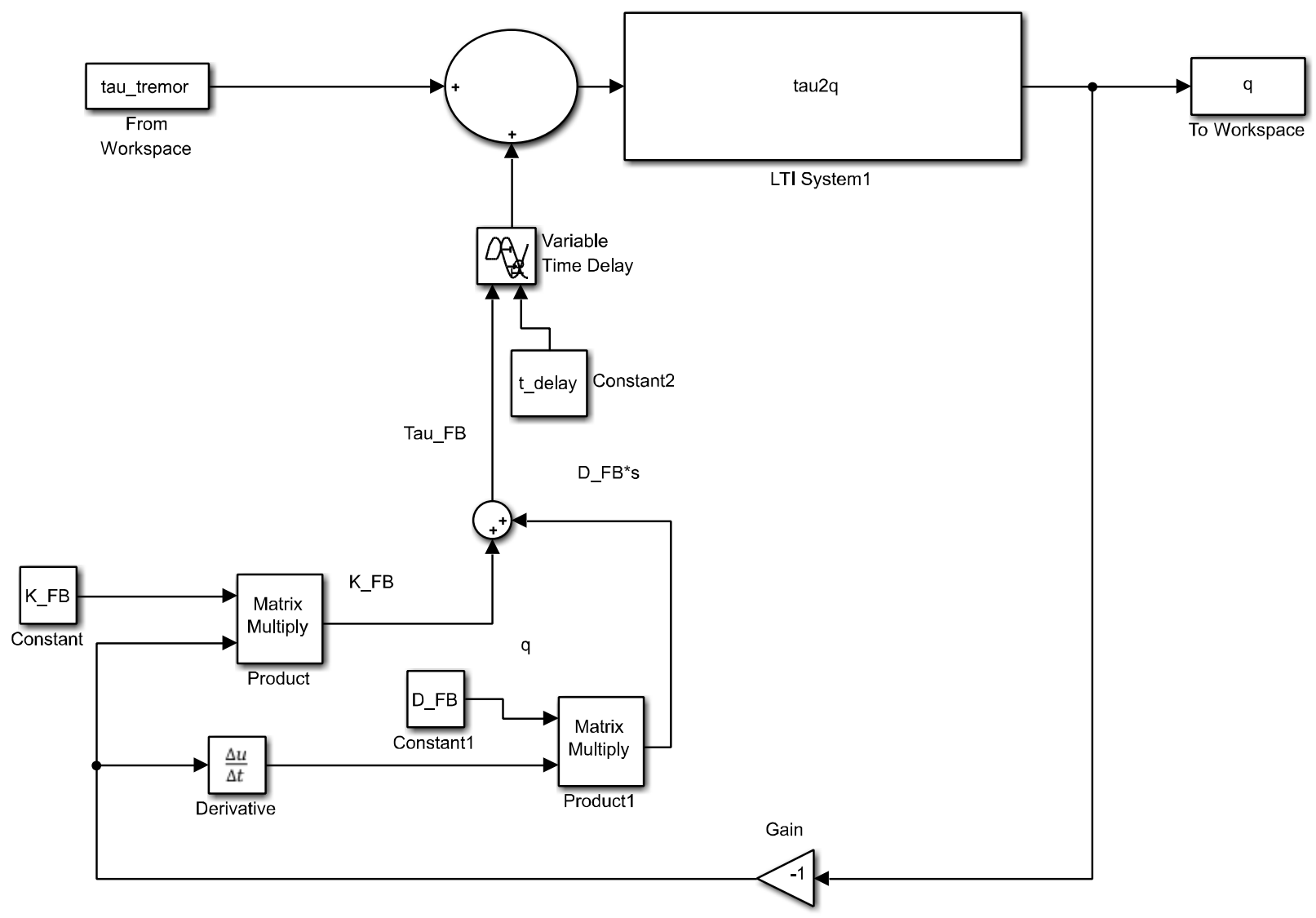

Figure 11. Simulink block diagram for DOF(torque)-level feedback. 
Homonymous and heteronymous muscular feedback relationships have been researched by multiple groups (see below). Unfortunately, many heteronymous gain values are unknown. From literature, we obtained gain values for approximately one half of the heteronymous inputoutput relationships (Table 3). Until the remaining blank values can be determined or inferred from trends, it will not be possible to model the heteronymous feedback of the upper-limb model.

Table 3. Heteronymous feedback gain values.

\begin{tabular}{|c|c|c|c|c|c|c|c|c|c|c|c|c|c|c|c|}
\hline & DELT1 & DELT2 & DELT3 & PECM2 & BIClong & BICshort & TRIlong & TRIlat & BRA & BRD & PT & FCR & FCU & ECR & ECU \\
\hline DELT1 & 1 & & & & & & & & & & & & & & \\
\hline DELT2 & & 1 & & & & & & & & & & & & & \\
\hline DELT3 & & & 1 & $-0.067^{2}$ & $0.019^{2}$ & $0.019^{2}$ & $0.032^{2}$ & $0.032^{2}$ & & & & & & & \\
\hline РECM2 & & & $-0.052^{2}$ & 1 & $0.009^{2}$ & $0.009^{2}$ & $0.022^{2}$ & $0.022^{2}$ & & & & & & & \\
\hline \multirow{2}{*}{ BIClong } & \multirow{2}{*}{$0.1^{1}$} & \multirow{2}{*}{$0.1^{1}$} & \multirow{2}{*}{$0.008^{2}$} & \multirow{2}{*}{$0.024^{2}$} & \multirow{2}{*}{$0.36^{3}$} & \multirow{2}{*}{$0.36^{3}$} & $-0.047^{2}$ & $-0.05^{2}$ & \multirow{2}{*}{$0.27^{3}$} & & $0.09^{3}$ & \multirow{2}{*}{$0^{1}$} & \multirow{2}{*}{$0^{1}$} & \multirow{2}{*}{$0^{1}$} & \multirow{2}{*}{$0^{1}$} \\
\hline & & & & & & & $0^{3}$ & $0^{3}$ & & & $-0.5^{4}$ & & & & \\
\hline \multirow{2}{*}{ BICshort } & \multirow{2}{*}{$0.1^{1}$} & \multirow{2}{*}{$0.1^{1}$} & \multirow{2}{*}{$0.008^{2}$} & \multirow{2}{*}{$0.024^{2}$} & \multirow{2}{*}{$0.36^{3}$} & \multirow{2}{*}{$0.36^{3}$} & $0^{3}$ & $0^{3}$ & \multirow{2}{*}{$0.27^{3}$} & & $0.09^{3}$ & \multirow{2}{*}{$0^{1}$} & \multirow{2}{*}{$0^{1}$} & \multirow{2}{*}{$0^{1}$} & \multirow{2}{*}{$0^{1}$} \\
\hline & & & & & & & $-0.047^{2}$ & $-0.05^{2}$ & & & $-0.5^{4}$ & & & & \\
\hline \multirow{3}{*}{ TRIlong } & \multirow{2}{*}{$-0.1^{1}$} & \multirow{2}{*}{$-0.1^{1}$} & $0.002^{2}$ & \multirow{3}{*}{$0.005^{2}$} & $-0.125^{2}$ & $-0.125^{2}$ & & & & & & & & & \\
\hline & & & $-0.1^{1}$ & & $0^{3}$ & $0^{3}$ & $1^{3}$ & $0.32^{3}$ & $0^{3}$ & & $0^{3}$ & $0^{1}$ & $0^{1}$ & $0^{1}$ & $-0.1^{1}$ \\
\hline & $-0.32^{5}$ & $-0.32^{5}$ & $-0.32^{5}$ & & 0 & 0 & & & & & & & & & \\
\hline TPUl & $01^{1}$ & $01^{1}$ & $0.002^{2}$ & $0005^{2}$ & $0^{3}$ & $0^{3}$ & $014^{3}$ & $041^{3}$ & $0^{3}$ & & $0^{3}$ & $0^{1}$ & $0^{1}$ & $0^{1}$ & 0.1 \\
\hline 1 & & & $-0.1^{1}$ & 0.000 & $-0.125^{2}$ & $-0.125^{2}$ & & & & & & & & & \\
\hline BRA & & & & & $0.41^{3}$ & $0.41^{3}$ & $0^{3}$ & $0^{3}$ & $0.73^{3}$ & & $0.41^{3}$ & & & & \\
\hline BRD & & & & & & & & & & 1 & $-0.55^{6}$ & $-0.4^{7}$ & & $0.5^{8}$ & \\
\hline DT & & & & & $0.091^{3}$ & $0.091^{3}$ & $0^{3}$ & $0^{3}$ & | & $\mid \begin{array}{lll}\mid & 2 & 10\end{array}$ & 0263 & & & & \\
\hline 11 & & & & & $0.36^{9}$ & $0.36^{9}$ & & & | & & 0.00 & & & & \\
\hline ECD & $-0.4^{1}$ & $-0.4^{1}$ & $-0.4^{1}$ & & $0.8^{11}$ & $0.8^{11}$ & $-0.59^{11}$ & $-0.59^{11}$ & & $\begin{array}{lll} & 0\end{array}$ & & $\log _{0} 1$ & $0.4^{12}$ & 021 & \\
\hline Fen & $-0.35^{5}$ & $-0.35^{5}$ & $-0.35^{5}$ & & $0.75^{1}$ & $0.75^{1}$ & $-0.1^{1}$ & $-0.1^{1}$ & & $\left.\right|^{-0.4}$ & & 0.00 & $0.6^{1}$ & $\mid-0.3$ & \\
\hline & م & م & $0^{1}$ & & & & & & & & & $0.2^{12}$ & & & \\
\hline FCU & & & $0.41^{13}$ & & $-0.1^{1}$ & $-0.1^{1}$ & $0^{1}$ & $0^{1}$ & & & & 1 & $0.3^{12}$ & & \\
\hline & $0^{5}$ & $0^{5}$ & $0^{5}$ & & & & & & & & & 0.2 & & & \\
\hline & 011 & $01^{1}$ & $-0.1^{1}$ & & $-0.83^{11}$ & $-0.83^{11}$ & $0.96^{11}$ & $0.96^{11}$ & & & & & & & \\
\hline ECR & & & $0.27^{13}$ & & $01^{1}$ & $01^{1}$ & 0 751 & $075^{1}$ & & $0.32^{8}$ & & $-0.3^{1}$ & & $0.4^{12}$ & $0^{12}$ \\
\hline & $-0.38^{5}$ & $-0.38^{5}$ & $-0.38^{5}$ & & -0.1 & -0.1 & 0.15 & 0.15 & & & & & & & \\
\hline ECU & & & & & & & & & & & & & & $0.2^{12}$ & $0.19^{12}$ \\
\hline
\end{tabular}


Legend for Table 3 citations: ${ }^{1}=[72],{ }^{2}=[73],{ }^{3}=[74],{ }^{4}=[75],{ }^{5}=[76],{ }^{6}=[77],{ }^{7}=$ $[78],{ }^{8}=[79],{ }^{9}=[80],{ }^{10}=[81],{ }^{11}=[82],{ }^{12}=[83],{ }^{13}=[84]$. Rows indicate the muscle the signal is coming from (afferents or "sensors"); columns are the muscle the signal is going to (efferents or "actuators"). Shaded diagonal cells are homonymous feedback. Values were taken from the frequency/probability of occurrence, except for in [74]. Negative vales denote inhibition; when both excitation and inhibition values were given, table value is excitationinhibition. When different values were found for the same cells, both are shown. Values were obtained from short latency, group I, studies and were normalized to have maximum value of unity.

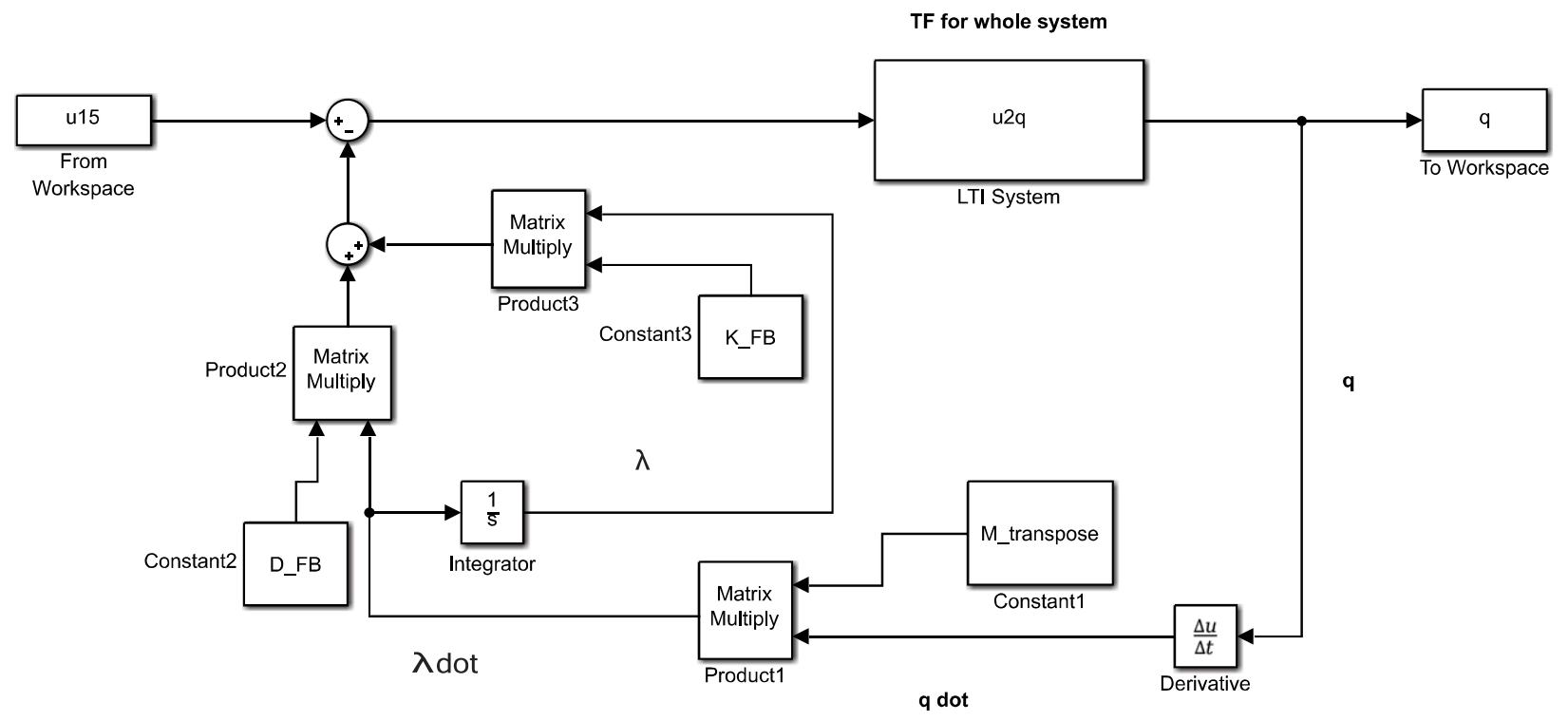

Figure 12. Simulink block diagram for muscle-level feedback 